\title{
Direct Quantification of Apparent Binding Indices from Quinidine-induced In Vivo Conduction Delay in Canine Myocardium
}

Frank N. Haugland, Susan B. Johnson, and Douglas L. Packer

Saint Mary's Hospital Complex, Mayo Foundation, Rochester, Minnesota 55902

\begin{abstract}
To characterize quantitatively the quinidine (QUIN)-induced conduction delay (CD) in vivo, canine ventricular activation times were examined with an epicardial mapping technique. $A$ high-resolution index of normalized (N) QUIN CD, derived from all 56 recording sites, was used to quantify QUIN effect. Repetitive stimulation elicited monoexponential increases in $\mathrm{CD}(\mathrm{N})$, the rates of which were a linear function of interpulse recovery interval, $t_{\mathbf{r}}$. Steady-state $\mathrm{CD}(\mathrm{N})$ was also linearly related to an exponential function of $t_{\mathrm{r}}$ and drug uptake rates. The frequency-dependent properties of QUIN in 14 dogs were characterized by apparent binding and unbinding rates of $k_{\mathrm{a}}$ $=7.1 \pm 3.5 \times 10^{6} \mathrm{M}^{-1} \mathrm{~s}^{-1}, l_{\mathrm{a}}=81 \pm 51 \mathrm{~s}^{-1}$ for activated, and $k_{\mathrm{r}}$ $=12.6 \pm 11.3 \times 10^{3} \mathrm{M}^{-1} \mathrm{~s}^{-1}, l_{\mathrm{r}}=0.51 \pm 0.26 \mathrm{~s}^{-1}$ for resting states. $k_{\mathrm{a}}$ and $l_{\mathrm{a}}$ were similar to values previously derived in canine Purkinje fibers. Drug unbinding at resting potentials was faster in vivo than previously observed in vitro. The time constant of recovery from QUIN block extracted from the interpulse recovery rate was also identical to that determined from post-mature stimulus diastolic scanning. As predicted by the two-state model, similar binding rates were also derived from declining $\mathrm{CD}(\mathrm{N})$ elicited by step decreases in heart rate. These findings represent a complete quantitative description of use-dependent QUIN CD in vivo and provide a firm foundation for characterizing antiarrhythmic drug action under physiologic and pathologic conditions. (J. Clin. Invest. 1994. 93:1798-1811.) Key words: antiarrhythmia agents • electromechanical coupling • electrophysiology • sodium channels • use dependence
\end{abstract}

\section{Introduction}

Class I antiarrhythmic drugs produce dynamic effects on the fast inward current through use-dependent block of $\mathrm{Na}^{+}$channels. Extensive in vitro analyses have described this phenomenon in terms of explicit mathematical models that yield fundamental rate constants accounting for the binding and unbinding of these drugs (1-3). Although the impact of antiar-

A preliminary report of these data was presented in abstract form at the 42nd Session of the American College of Cardiology, Anaheim, CA, March 1993.

Address reprint requests to Dr. Douglas L. Packer, 4-416 Alfred Building, Saint Mary's Hospital Complex, Mayo Foundation, Rochester, MN 55902.

Received for publication 3 August 1993 and in revised form 11 November 1993.

J. Clin. Invest.

(c) The American Society for Clinical Investigation, Inc.

0021-9738/94/04/1798/14 \$2.00

Volume 93, April 1994, 1798-1811 rhythmic agents on $\mathrm{Na}^{+}$current $\left(l_{\mathrm{Na}}\right)^{1}$ and maximal upstroke velocity of the action potential $\left(V_{\max }\right)$ has been extensively studied in cellular preparations, their dynamic effects on myocardial excitation and conduction in vivo have been less completely characterized.

Any quantitatively accurate characterization of drug-induced use-dependent effects in propagating systems is critically dependent upon demonstrable changes in measures of conduction, which should be related to events occurring at the channel level. Although the exact relationship between $\mathrm{Na}^{+}$conductance $\left(g_{\mathrm{Na}}\right)$ and conduction velocity $(\theta)$ is uncertain, several investigators have demonstrated a linear relationship between drug-induced changes in $\theta^{2}$ and $V_{\max }$ in guinea pig papillary muscle (4) and canine Purkinje fibers (5). Furthermore, assuming a linear relationship between $g_{\mathrm{Na}}$ and $\theta^{2}$, Nattel (6) estimated the time constant of recovery $\left(\tau_{\mathrm{r}}\right)$ from lidocaine block from measures of conduction in canine Purkinje fibers and found it to be quantitatively similar to that derived from an analysis of $V_{\max }$. In a subsequent study, Packer et al. (5) used a quantitative approach based on a two-state model of the quinidine (QUIN)- $\mathrm{Na}^{+}$channel interaction to estimate four fundamental rate constants of forward and reverse drug binding. Comparable information was obtained from both $\theta^{2}$ and $V_{\max }$, suggesting that changes in measures of in vitro conduction due to drug reflect changes occurring at the membrane level.

In contrast to the extensive literature detailing these use-dependent conduction changes in vitro, the analysis of use dependence in vivo has been largely qualitative. Several investigators have noted progressive prolongation of the QRS duration or the $\mathrm{H}-\mathrm{V}$ interval with repetitive stimulation in the presence of a variety of antiarrhythmic agents in both animals and man (711). Nevertheless, specific effects of these drugs have been less well characterized quantitatively. Davis et al. (12) derived $\tau_{\mathrm{r}}$ from lidocaine block from the $\mathrm{H}-\mathrm{V}$ interval in anesthetized dogs. Bajaj et al. (13) also derived $\tau_{\mathrm{r}}$ for mexiletine block from canine ventricular activation using an epicardial electrode array. Physiologic heart rates precluded a quantification of the relatively long time constants describing QUIN recovery kinetics. In a more recent analysis, Nattel and Jing (14) estimated $\tau_{\mathrm{r}}$ for procainamide recovery in the intact canine heart. In addition, in a study from the same institution, Villemaire et al. (15) used a theoretical framework developed by Starmer and Grant (16) and Starmer et al. (17) to estimate fundamental rate constants describing procainamide action in the intact heart. The latter study, however, relied exclusively on total epicardial acti-

1. Abbreviations used in this paper: APD, action potential duration; $\mathrm{A}-\mathrm{V}$, atrioventricular; BEG, bipolar electrogram; CD, conduction delay; $\mathrm{CD}(\mathrm{N})$, normalized conduction delay; $g_{\mathrm{Na}}, \mathrm{Na}^{+}$conductance; $\mathrm{I}_{\mathrm{Na}}$, $\mathrm{Na}^{+}$current; ISI, interstimulus interval; MAP, monophasic action potential; QUIN, quinidine; $V_{\max }$, maximal upstroke velocity of the action potential; $\theta$, conduction velocity; $\tau_{\mathrm{r}}$, time constant of recovery from block. 
vation time of the canine heart and was restricted to a description of procainamide binding.

Complete quantitative information detailing both steadystate and kinetic properties of QUIN effect in vivo has not been available. The present study was, therefore, undertaken to $(a)$ develop a quantitatively accurate description of QUIN effect in terms of apparent binding and unbinding rates derived from use-dependent conduction delay, $(b)$ determine whether sufficient quantitative information can be derived from a restricted range of physiologic heart rates to provide comparable quantitative descriptions, and $(c)$ assess the impact of changes in electrical-mechanical coupling accompanying atrioventricular (AV) dissociation on the quantitative analysis of antiarrhythmic drug action in vivo.

\section{Methods}

General. All studies were performed using the intact canine heart. The methods utilized were reviewed and approved by the Mayo Foundation Institutional Animal Care and Use Committee. Studies were performed on $18-25-\mathrm{kg}$ animals after the administration of $30 \mathrm{mg} / \mathrm{kg}$ of intravenous pentobarbital delivered through an 18-gauge indwelling teflon catheter inserted into the right forearm. The animals were intubated with an endotracheal tube and ventilated using a Harvard Apparatus (S. Natick, MA) pump with tidal volumes derived from a nomogram relating animal size and arterial $\mathrm{pH}$. Monitoring of seven surface electrocardiograph leads was instituted. Subsequently, anesthesia was maintained by intermittent intravenous bolus administration of pentobarbital at an average rate of $1.5 \mathrm{mg} / \mathrm{kg} \cdot \mathrm{h}$ with additional supplementation as needed. A second 18-gauge indwelling teflon catheter was inserted in the left forearm for drug administration. A 7 French teflon sheath was introduced into the right femoral artery for blood pressure, serial blood gas, and electrolyte monitoring. In each experiment, a 7 French balloon-tipped catheter was introduced via the right femoral artery sheath and advanced $18-20 \mathrm{~cm}$ to a level immediately distal to the renal arteries. In most experiments, this balloon was subsequently utilized to maintain blood pressure at a minimum level of $90 / 50 \mathrm{~mm}$ in the eventuality of QUIN-induced hypotension. A median sternotomy was then performed and a pericardial cradle created. Bipolar electrodes for both pacing and recording were sutured near the sinus node and the acute margin of the right ventricle near the $\mathrm{A}-\mathrm{V}$ groove. To facilitate an examination of drug effects at a wide range of heart rates, complete heart block was created by injecting $0.2-0.3 \mathrm{ml}$ of $37 \%$ formalin immediately adjacent to the noncoronary cusp of the aorta (18). To eliminate any confounding effects of changes in autonomic tone on QUIN binding during the course of the study, the cervical vagal trunks and ansae subclaviae were isolated, doubly ligated, and transected. The bilateral vagotomy was also used to limit any potential confounding vagolytic effect of pentobarbital anesthesia (19). Myocardial temperature, monitored using a septal probe positioned near the apex of the left ventricle, was maintained at $37^{\circ} \mathrm{C}$ with a circulating water flow heating pad, overhead heatlamps, and apposition of the chest wall.

Recording techniques. Myocardial activation under control and experimental conditions was examined using an epicardial sock mapping technique. A sock, made of nylon mesh, containing 56 bipolar electrodes (intra-bipolar pair spacing of $2 \mathrm{~mm}$ ) was secured over the left and right ventricles with six to eight 3-0 silk sutures placed along the A-V ring. In addition, the apical region of the sock was anchored with a single 3-0 silk suture which also secured the temperature probe for continuous myocardial temperature monitoring. Bipolar recordings were made using either a Bard model 64 mapping system (Bard Electrophysiology, Billerica, MA; initial five experiments) or a Cardiomapp 255 mapping system (Arrhythmia Research Technology [ART], Austin, TX; subsequent nine experiments). The Bard system acquired and digitally converted $8 \mathrm{~s}$ of continuous bipolar analog signals at a $1.0-\mathrm{kHz}$ sampling frequency with 12-bit accuracy. The ART mapping system acquired $27 \mathrm{~s}$ of continuous bipolar analog signals with 12 bit analog to digital conversion at a $0.98-\mathrm{kHz}$ sampling frequency.

Determination of action potential duration (APD). In all experiments, an epicardial monophasic action potential (MAP) probe (EP Technologies, Sunnyvale, CA) was mounted in a spring-loaded custom-designed frame and positioned along the anterior left ventricle near the apex to measure changes in APD at 50\%, 75\%, 90\%, and 95\% repolarization. In seven cases, a 6 French MAP catheter was also introduced into the left ventricle via a left atriotomy, for examination of endocardial APD. Constant contact pressure was maintained during all MAP recordings. $\mathrm{APD}_{50}, \mathrm{APD}_{75}, \mathrm{APD}_{90}$, and $\mathrm{APD}_{95}$ were determined off-line using custom-designed software and the interpulse recovery time, $t_{\mathrm{r}}$, was calculated as interstimulus interval (ISI)-APD 90 . Auxiliary channels of the mapping system were modified to allow direct current recordings of MAPs and stimulator synchronization signals for a measurement reference.

Stimulation protocols. Pulse train stimulation was performed using a Bloom BERS 420 stimulator (Bloom Associates, Reading, PA) externally triggered by specially designed software maintained on an IBM personal computer. Under baseline conditions, the right ventricle was paced at an ISI of $1 \mathrm{~s}$ at twice diastolic threshold with pulses of $2 \mathrm{~ms}$ in duration. In the initial five experiments, data were acquired under control and drug conditions at the baseline ISI of $1.0 \mathrm{~s}$ and after $40 \mathrm{~s}$ of constant pacing at ISIs of $0.75,0.6,0.45$, and $0.3 \mathrm{~s}$. Because of limitations imposed by the 8-s acquisition window, data for the first $12 \mathrm{~s}$ and final $8 \mathrm{~s}$ of $40 \mathrm{~s}$ pulse trains at ISI $=0.75,0.60,0.45$, and $0.30 \mathrm{~s}$ were reconstructed from three window acquisitions during successive trains at each ISI. The ventricle was paced for $40 \mathrm{~s}$ at an ISI $=1.0 \mathrm{~s}$ between the respective pulse trains for recovery from block.

In the subsequent nine experiments, 27-s continuous recordings were made with the ART mapping system. Pacing between pulse trains was also increased to $70 \mathrm{~s}$ at the baseline cycle length $(1.0 \mathrm{~s})$ to allow transfer of data to the hard disk. Since the initial studies showed that steady-state block was universally reached in $<12 \mathrm{~s}$, activation data were acquired with 12 -s pulse trains at ISIs of $0.75,0.60,0.45$, and 0.30 s. There were no statistically significant differences between data obtained using the two data acquisition systems. The effect of A-V synchrony observed in the first 10 studies was eliminated in the final four experiments through $A-V$ sequential pacing with an A-V delay of $100 \mathrm{~ms}$ at each pacing rate.

Drug infusions. QUIN was administered in a loading dose of 1.02 $\mathrm{mg} / \mathrm{kg} \cdot \mathrm{min}$ given over $22 \mathrm{~min}$ followed by a maintenance infusion at $0.15 \mathrm{mg} / \mathrm{kg} \cdot \min$. The serum QUIN concentrations were determined at the Mayo Foundation drug assay laboratory using an enzyme-mediated immunoassay technique which was accurate to $\pm 5 \%$. QUIN levels were $5.6 \pm 1.5 \mu \mathrm{g} / \mathrm{ml}$ (range $3.8-8.2 \mu \mathrm{g} / \mathrm{ml}$ ) immediately after completion of the bolus, $4.3 \pm 0.7 \mu \mathrm{g} / \mathrm{ml}$ (range $3.2-6.4 \mu \mathrm{g} / \mathrm{ml}$ ) $15 \mathrm{~min}$ after initiation of the maintenance infusion, and $4.3 \pm 0.5 \mu \mathrm{g} / \mathrm{ml}$ (range $3.2-5.0 \mu \mathrm{g} / \mathrm{ml}$ ) upon completion of experimental data acquisition. The calculated average serum concentration during data acquisition was $4.3 \pm 0.5 \mu \mathrm{g} / \mathrm{ml}$ (range $3.6-5.7 \mu \mathrm{g} / \mathrm{ml}$ ).

Off-line data analysis. Raw data files generated by acquisition hardware were transferred via an RS-232 serial interface (Bard) or an ethernet link (ART) to a Macintosh IIci personal computer and analyzed using custom-made software (written by Dr. Haugland). This software allowed a straightforward review of desired bipolar electrograms (BEGs) as well as automated activation time detection of each beat within each raw data file. The electrograms were displayed in panels of eight tracings to facilitate overreading. Specific panels from the same sequential beat of a train from control and drug states or successive beats of a given train were displayed side by side. The peak amplitude of monophasic waves and the peak of the largest deflection of triphasic waves were taken as the local activation time. The maximum derivative of the first or largest rapid deflection (typically near the crossing point of the isoelectric line) was used as the local activation time in the case of biphasic or complex wave forms.

Activation time determination was not dependent on a "global" command mandating a specific method of activation time determina- 
tion for all electrograms. Rather, system flexibility permitted individual selection of a specific detection algorithm for consistent use in determining activation times for a given electrode over the course of a study. This consistency of activation time determination was also facilitated by the ability to superimpose electrograms from any reference beat and successive beats under analysis. Electrograms were deleted from analysis if $(a)$ the amplitude was $<0.25 \mathrm{mV}$ or $(b)$ on successive beats within a single data acquisition, an identified waveform became obscured by the appearance of $60 \mathrm{~Hz}$ noise, with or without large slow waves, or high frequency spikes which saturated the analog-to-digital converters. For each experiment, at least 226 heartbeats $(12,656$ electrograms) were analyzed. Activation maps were generated by returning the activation times from each electrode to the acquisition platform electrode template, and isochronal lines were drawn at 10-ms intervals based on local depolarization times.

Custom software was also used to plot activation times from each of the 56 electrodes during a specific beat in the presence of QUIN as a function of activation time during an analogous beat under control conditions. Each point of such a plot represents the relative conduction delay (CD) due to QUIN at a specific electrode site for a given pulse. In turn, the slope of the line generated from the plot of the equally weighted relative $C D s$ at each of the 56 electrodes reflects the normalized $(\mathrm{N})$ drug-induced $\mathrm{CD}, \mathrm{CD}(\mathrm{N})$, over the entire heart for that pulse. Increased block generated by faster pacing rates, or with each successive pulse of a pulse train, produced an incremental slope change of subsequent plots. A nonlinear least squares exponential fitting procedure was utilized to describe the time course of change in incremental $\mathrm{CD}(\mathrm{N})$ or block accumulation reflected by slope change occurring over the pulse train.

In addition to describing fractional block, the plots of $\mathrm{CD}(\mathrm{N})$ provided a rapid quantitative assessment of the uniformity of global ventricular activation under control and test conditions. Consistency of activation pattern was indicated by linearity of the relationship between activation times under drug and control conditions (14). Furthermore, consistency of activation sequence was indicated qualitatively by similarities in isochronal activation patterns observed on the epicardial maps (14).

Experimental calculation of fractional block. The estimation of fractional QUIN-induced $\mathrm{Na}^{+}$channel block from changes in conduction is based on assumptions previously applied and validated by Packer et al. (5) in vitro and by Nattel and coworkers in vivo $(14,15)$. It was assumed that $\theta^{2}$ was a linear function of $g_{\mathrm{Na}}$ :

$\theta^{2} \propto g_{\mathrm{Na}}$

and that small changes in $\theta^{2}$ due to drug accurately reflected changes in $\mathrm{Na}^{+}$channel block:

$\mathrm{d} \theta^{2} \propto \mathrm{d} g_{\mathrm{Na}}$

Estimated fractional block, $b_{n}$, during pulse train stimulation, from $\theta^{2}$, was expressed as:

$b_{\mathrm{n}}=1-\left(\theta_{\mathrm{d}}^{2} / \theta_{\mathrm{c}}^{2}\right)$

where $\theta_{\mathrm{d}}^{2}$ is the squared conduction velocity for a given action potential in the presence of drug and $\theta_{\mathrm{c}}^{2}$ is the squared conduction velocity under control conditions.

In the intact heart, absolute conduction velocities may be difficult to determine given the limitations in specifying the conduction pathway between two recording sites. Nattel and coworkers showed, however, that when the activation sequence and, therefore, conduction pathway under control and experimental conditions are constant, relative conduction velocities may be estimated in vivo by comparing conduction times under control and experimental conditions $(14,15)$. $\theta^{2}(\mathrm{~N})$ was therefore derived using available data generated under control and drug conditions for each heartbeat:

$\theta^{2}(\mathrm{~N}) \approx \theta_{\mathrm{d}}^{2} / \theta_{\mathrm{c}}^{2}$ where block was viewed as:

$b_{\mathrm{n}} \approx 1-\theta^{2}(\mathrm{~N})$

In this fashion, the apparent fractional $\mathrm{Na}^{+}$channel block $\left(b_{n}\right)$ in vivo by QUIN was calculated for all heartbeats under both steady-state and dynamic conditions. To allow additional comparisons with previously published in vitro data (5), calculations were also performed using changes in $\mathrm{CD}(\mathrm{N})$ as the index of block.

Apparent binding rate determination. The normalized slope expressions of $C D(N)$ were used as markers of drug effect under both steadystate and dynamic conditions. From these, the kinetics of accumulation of and recovery from block were described in terms of a special application of a three-state model of drug binding $(5,20)$. For this approach, overall drug block is viewed as an alternating sequence of three exponentials describing drug uptake during active, inactive, and resting channel states of duration $t_{\mathrm{a}}, t_{\mathrm{i}}$, and $t_{\mathrm{r}}$, with descriptive drug uptake rates of $\lambda_{a}, \lambda_{i}$, and $\lambda_{r}$, respectively. Here the overall drug uptake rate for net drug binding is:

$\lambda^{*}=\lambda_{\mathrm{a}} t_{\mathrm{a}}+\lambda_{\mathrm{i}} t_{\mathrm{i}}+\lambda_{\mathrm{r}} t_{\mathrm{r}}$

Under conditions where both pulse duration and recovery times can be manipulated through voltage clamp (20) or pharmacologic alterations (21), six parameters describing drug binding and unbinding, in terms of forward $\left(k_{\mathrm{a}}, k_{\mathrm{i}}, k_{\mathrm{r}}\right)$ and reverse $\left(l_{\mathrm{a}}, l_{\mathrm{i}}, l_{\mathrm{r}}\right)$ apparent binding rate constants for each of the three states can be derived (20). Under conditions where APD cannot be manipulated, such as during these in vivo studies, channel binding was viewed as a two-state process: $(a)$ an accessible state, the duration of which is the mean channel open time, $t_{\mathrm{a}}$, and $(b) \mathrm{a}$ recovery state which is related to the interstimulus interval (5). For drug studies using "open-channel blocking" agents such as QUIN (2224) or flecainide (25) without inactivated state block, such assumptions are reasonable, particularly since recent studies have suggested that the mean channel open time does not change appreciably with drug blockade of $\mathrm{Na}^{+}$channels $(26,27)$. Under these circumstances, the overall drug uptake rate was given as the weighted sum of the two state-dependent drug uptake rates $\lambda_{\mathrm{a}}$ and $\lambda_{\mathrm{r}}$, where:

$\lambda^{*}=\lambda_{\mathrm{a}} t_{\mathrm{a}}+\lambda_{\mathrm{r}} t_{\mathrm{r}}$

This suggests that a linear relationship exists between overall drug uptake rate, $\lambda^{*}$ obtained from the exponential fit to the $C D$-pulse number relationship and the interpulse recovery interval, $t_{\mathrm{r}}$. From this relationship, the activated state uptake rate, $\lambda_{\mathrm{a}}$, was derived from the $y$-intercept, and the recovery rate, $\lambda_{r}$, was obtained from the slope.

This model also predicts a second linear relationship between steady-state blockade, $r_{\text {ss }}$, and an exponential expression $\gamma$, where:

$\gamma=\left(1-e^{-\lambda_{r r}}\right) /\left(1-e^{-\lambda^{*}}\right)$

From this second relationship, the equilibrium expression describing block during the activated state, $a_{\infty}$, was derived from the $y$-intercept, while the resting state expression $r_{\infty}$ was derived from the slope:

$r_{\mathrm{ss}}=\left(a_{\infty}-r_{\infty}\right) \gamma+a_{\infty}$

Here, $r_{\infty}$ represents tonic block. From $\lambda_{\mathrm{a}}, \lambda_{\mathrm{r}}, a_{\infty}$, and $r_{\infty}$, the four fundamental apparent binding rate constants can be derived from simultaneous solution of the following four expressions:

$$
\begin{aligned}
\lambda_{\mathrm{a}} & =k_{\mathrm{a}} D+l_{\mathrm{a}} \\
\lambda_{\mathrm{r}} & =k_{\mathrm{r}} D+l_{\mathrm{r}} \\
a_{\infty} & =k_{\mathrm{a}} D /\left(k_{\mathrm{a}} D+l_{\mathrm{a}}\right) \\
r_{\infty} & =k_{\mathrm{r}} D /\left(k_{\mathrm{r}} D+l_{\mathrm{r}}\right)
\end{aligned}
$$

These apparent binding rates, in turn, were used to reflect drug effect at the channel level, as derived from changes in incremental conduction. At a minimum, these served as a useful index of QUIN binding, even in the presence of distorted relationships between $g_{\mathrm{Na}}, V_{\max }$, and $\theta^{2}$. The analytic methods of the parameter estimation procedures and their 
specific implementation in the quantification of QUIN-induced CD have been described previously in greater detail (5).

Statistic analysis. All experimental results are reported as mean \pm SD. Data acquired under control and experimental conditions were compared using two-tailed $t$ tests with $P<0.05$ taken as significant. Additional comparisons using paired $t$ tests are identified in the Results. Because there were no statistically significant differences between the initial five and the final nine experiments using the two data acquisition systems, the data were pooled for statistical comparisons.

\section{Results}

Analysis of ventricular activation under control conditions. Ventricular activation was examined under control conditions at ISIs $=1.0-0.3 \mathrm{~s}$. Activation spread from the right ventricular pacing site near the $A-V$ groove to the lateral left ventricle with representative epicardial BEGs over the activation course as shown in Fig. $1 A$. Corresponding activation maps with isochronal lines drawn at 10 -ms intervals, as illustrated in Fig. $1 \mathrm{~B}$, showed no qualitative change in activation sequence or ventricular activation times with progressively faster pacing rates from ISIs $=1.0-0.3 \mathrm{~s}$. The frequency independence of control ventricular activation times at these heart rates was reflected in the similarity in activation maps obtained at all ISIs $\geq 0.3 \mathrm{~s}$. In three additional experiments, control activation maps obtained at ISI $=0.2 \mathrm{~s}$ showed a similar activation sequence as observed at ISI $=1.0 \mathrm{~s}$ but ventricular activation times were modestly prolonged by $1-3 \%$.

MAPs under control conditions. MAPs were recorded under control conditions from the apical epicardial surface of the left ventricle and from the $\mathrm{LV}$ endocardium. Control $\mathrm{APD}_{90}$ 's showed a progressive rate-dependent decline in repolarization time at both endocardial and epicardial sites. At ISI $=1.0 \mathrm{~s}$, control epicardial $\mathrm{APD}_{90}$ was $212 \pm 19 \mathrm{~ms}(n=14)$, whereas at ISI $=0.3 \mathrm{~s}$, control $\mathrm{APD}_{90}$ shortened to $172 \pm 19(P=0.0001$, paired $t$ test). Epicardial action potentials had a "spike and dome" morphology at all pacing rates, with less evident notching at shorter ISIs. Comparisons between endocardial and epicardial recording sites in three experiments showed that endocardial APDs were typically 10-30 ms longer, had a longer plateau phase, and less spike and dome appearance than epicardial APDs.

Quantification of steady-state QUIN-induced conduction delay in vivo. The quantitative and qualitative effects of QUIN on ventricular activation times were examined at ISIs of 1.0$0.3 \mathrm{~s}$. At the slowest pacing rate, demonstrable QUIN-induced delays in activation times were observed, with the largest absolute prolongations noted at the electrodes furthest from the pacing site. As shown in Fig. 2, activation at the electrode closest to the pacing site (BEG G5) during pacing at an ISI $=1.0 \mathrm{~s}$ was delayed by $3 \mathrm{~ms}$ from that seen under control conditions, while the activation at BEG D2 was delayed by 18 ms (Figs. $2 A$ vs. $1 A$ ). Activation maps as shown in Fig. $2 B$ indicated no qualitative change in the sequence of ventricular activation with drug, although isochrones were more densely crowded with QUIN than under control conditions (Fig. $1 B$ ), reflecting global slowing of conduction due to drug.

Activation maps prepared from each of 14 experiments showed that, in the presence of QUIN, steady-state activation times were always rate dependent, with the largest conduction delays occurring at the fastest heart rates. At each pacing rate,
A

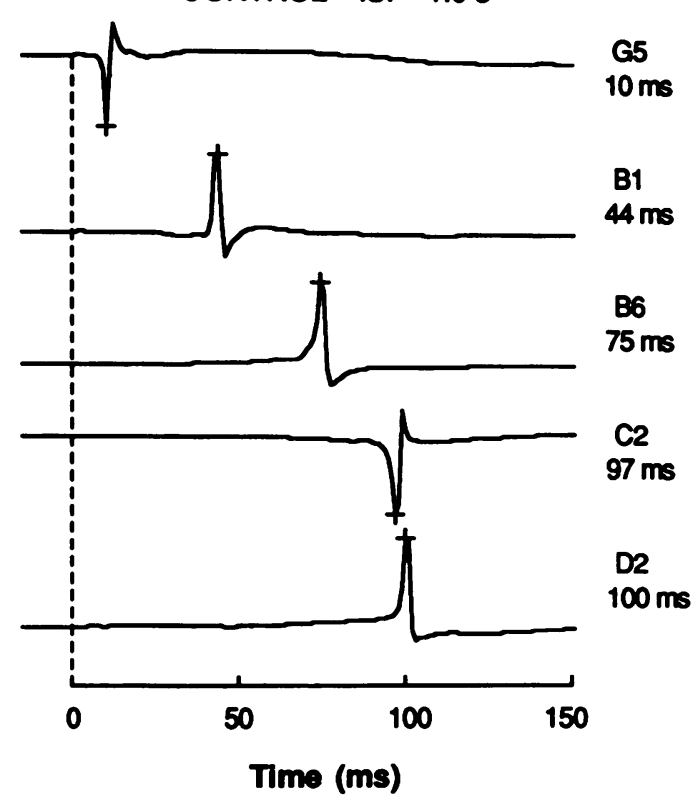

B

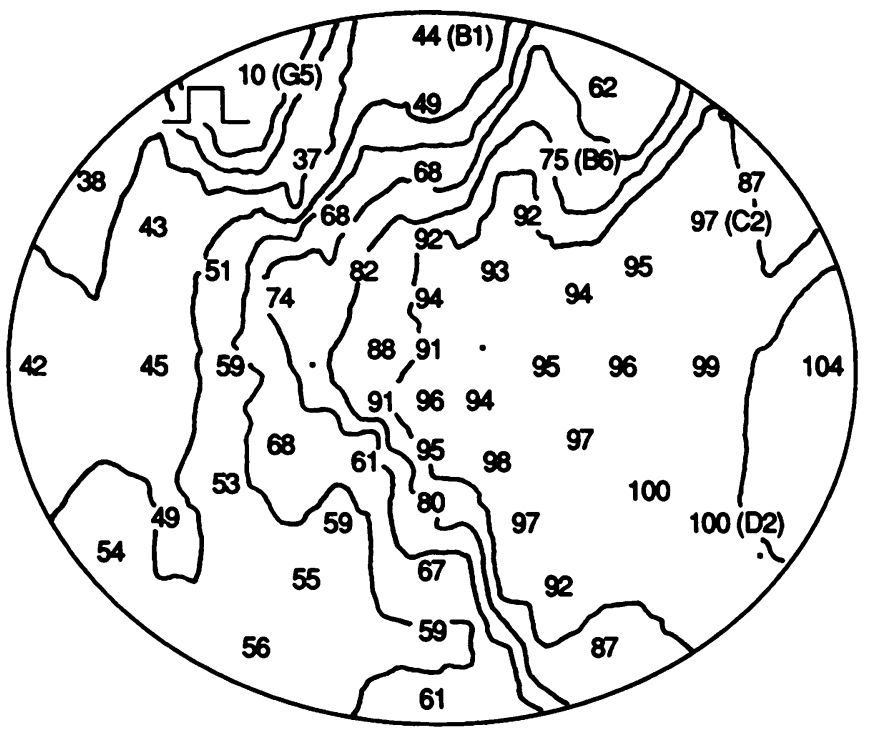

Figure 1. Ventricular activation of the intact canine heart under control conditions at an ISI of $1.0 \mathrm{~s}$. $(A)$ Representative BEGs along the path of ventricular activation. The top trace shows BEG G5, which was closest to the right ventricular pacing site. The time of the local activation as indicated by the cursor $(+)$ was $10 \mathrm{~ms}$. In this and all subsequent figures, the activation times are given relative to the onset of the 2-ms ventricular stimulus (see Methods). The successive traces show recordings with increasing activation times as excitation spread across the anterior myocardial surface. The bottom trace shows BEG D2 located on the posterior left ventricle, which was activated 100 ms from the onset of the ventricular stimulus. $(B)$ The corresponding activation map for the same heartbeat as shown in $A$. The numbers indicate the activation times (in ms) determined for each BEG. The solid lines indicate activation isochrones which were drawn at $10-\mathrm{ms}$ intervals relative to the point of earliest activation (BEG G5). 

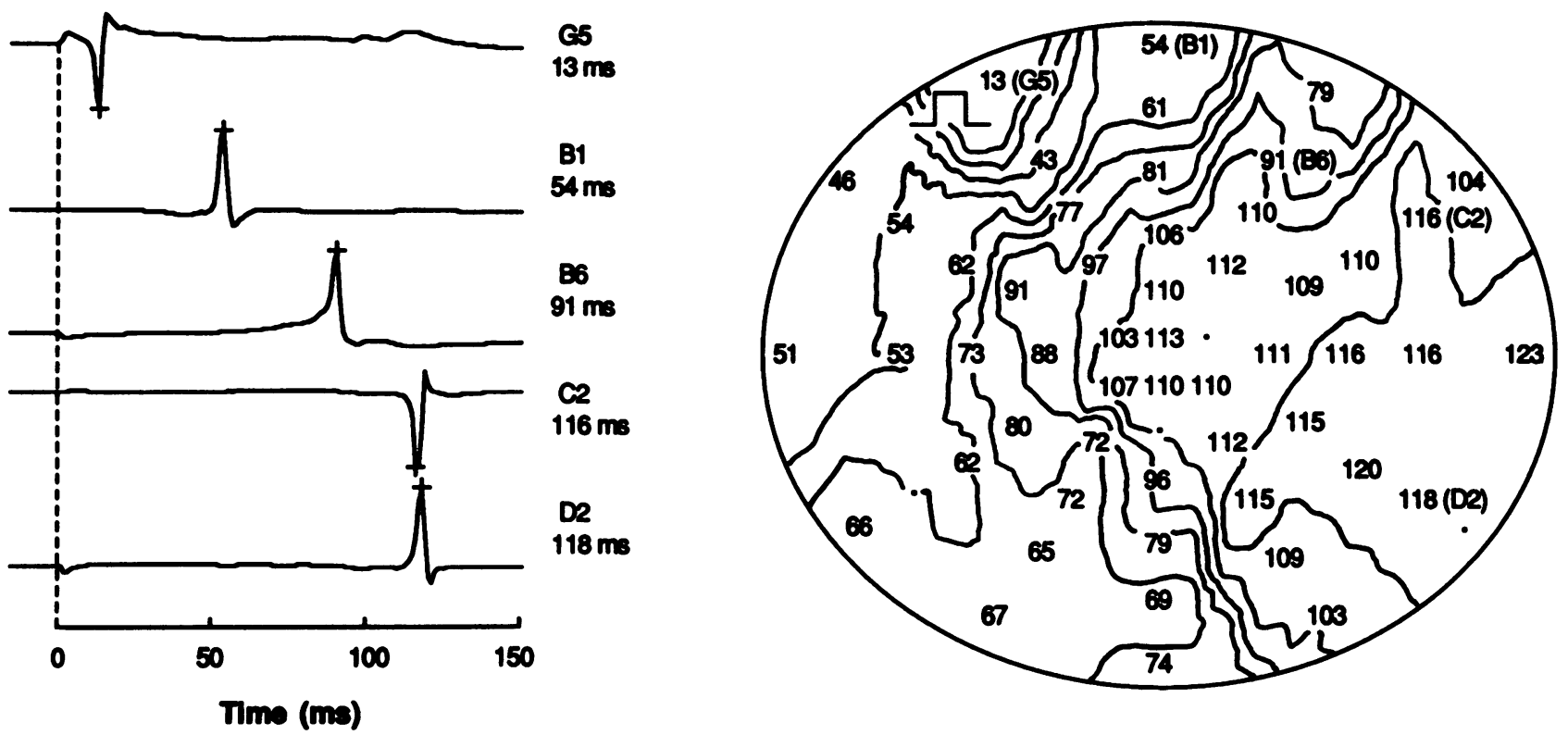

Figure 2. Ventricular activation mapping in the presence of QUIN at an ISI of $1.0 \mathrm{~s}$ from the same experiment as shown in Fig. 1. $(A)$ Representative BEGs recorded from the same sites as shown under control conditions. In comparison to control conditions (Fig. $1 \mathrm{~A}$ ), the activation times are prolonged, and the magnitude of the delay varies according to the location of the BEG. $(B)$ The corresponding activation map for the same heartbeat as shown in $A$. In comparison to control (Fig. $1 \mathrm{~B}$ ), the shape of the isochrones is qualitatively similar but the spacing is crowded indicating global slowing of the activation wavefront without gross alteration in the sequence of ventricular activation.

the new steady state was reached within $12 \mathrm{~s}$. As exemplified in Fig. 3, after $12 \mathrm{~s}$ of constant pacing at an ISI $=0.3 \mathrm{~s}$, isochrone patterns under control conditions (Fig. $3 A$ ) and with QUIN (Fig. $3 B$ ) were again qualitatively similar, indicating that the macroscopic sequence of ventricular activation did not change.

To obtain a quantitative index of global drug-induced CD as a reflection of use-dependent $\mathrm{Na}^{+}$channel block, the steady- state activation times at a given pacing rate disclosed at each of 56 recording sites measured in the presence of QUIN were plotted as a function of the activation times measured from the same electrodes under control conditions. As shown in Fig. 4 $A$, each point on the plot represented the CD at one of 56 electrodes, while the slope of this relationship provided a global index of normalized drug-induced $C D[C D(N)]$ over the epi-
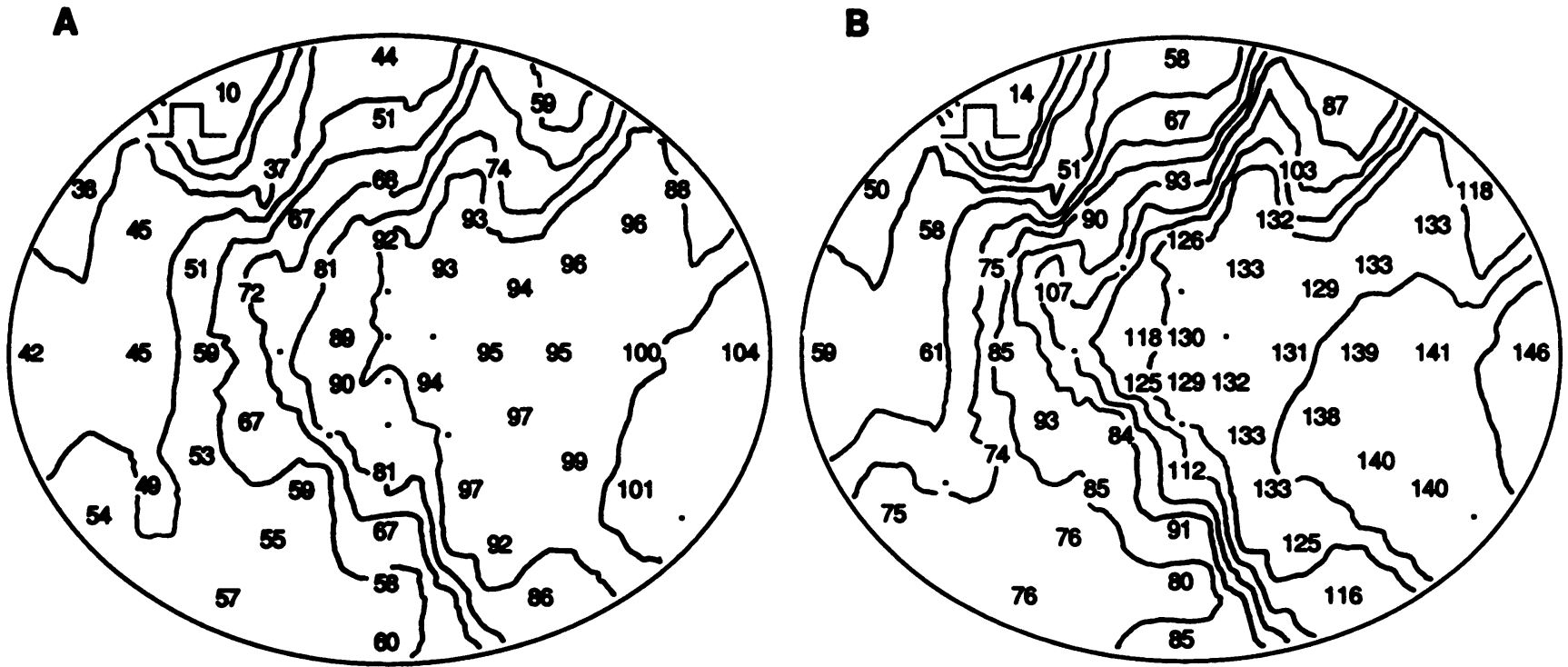

Figure 3. Further slowing of ventricular activation by QUIN at an ISI $=0.3 \mathrm{~s} .(A)$ Activation map recorded under control conditions. This map is nearly identical to the control data at ISI $=1.0 \mathrm{~s}$ (Fig. $1 B$ ). $(B)$ Activation map recorded after the administration of QUIN. Isochrones are more crowded than observed with QUIN at ISI $=1.0 \mathrm{~s}$ (Fig. $2 B$ ). 

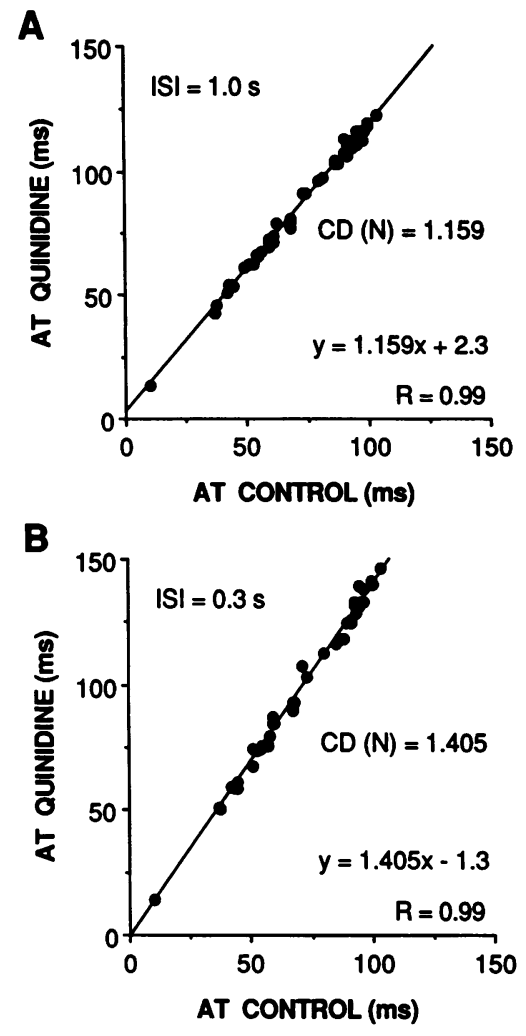

Figure 4. Quantification of steady state QUINinduced conduction delay in vivo. $(A)$ The normalized conduction delay $[C D(N)]$ with QUIN at ISI $=1.0 \mathrm{~s}$ is defined as the slope of the relationship (1.159) between activation times (ATs) in the presence of QUIN (vertical axis) plotted as a function of the ATs from the same BEG sites under control conditions (horizontal axis). The data fell on a straight line, indicating constancy of activation sequence. $(B)$ The comparably derived $\mathrm{CD}(\mathrm{N})$ with QUIN at ISI $=0.3$ $\mathbf{s}$ derived from activation times in Fig. 3. The data fell on a straight line with a $C D(N)$ $=1.405$.

cardial surface for a given beat. In Fig. $4 A$, the slope of $1.159(r$ $=0.99$ ) reflects a $15.9 \%$ increase in $\mathrm{CD}(\mathrm{N})$ at steady state during pacing at an ISI $=1.0 \mathrm{~s}$.

In all experiments, frequency-dependent block by QUIN was manifested by progressive steepening of the slope of the relationship between drug and control activation times with the greatest $\mathrm{CD}(\mathrm{N})$ occurring at the shortest cycle lengths. As shown in Fig. $4 B, C D(N)$ after $12 \mathrm{~s}$ of pacing at ISI $=0.3 \mathrm{~s}$ was 1.405 , indicating a $40.5 \%$ prolongation of global conduction time. As shown in Fig. $5 A$, the average steady-state $\mathrm{CD}(\mathrm{N})$ in 14 animals ranged from $1.147 \pm 0.079$ at ISI $=1.0 \mathrm{~s}$ to $1.325 \pm 0.130$ at ISI $=0.3 \mathrm{~s}(P<0.0003)$. A routine assessment of $\mathrm{CD}(\mathrm{N})$ at an ISI $=0.2 \mathrm{~s}$ was not possible due to intermittent capture with pacing at this rate. Nevertheless, in one additional experiment, pacing with an exponentially declining ISI was used to maintain ventricular capture. With this method, the $\mathrm{CD}(\mathrm{N})$ at an ISI $=0.2 \mathrm{~s}$ was found to be 1.639 . In all experiments, the "normalized conduction velocity" $[\theta(\mathrm{N})]$, defined as the simple reciprocal of $\mathrm{CD}(\mathrm{N})$, and the "squared normalized conduction velocity" $\left[\theta^{2}(\mathrm{~N})\right]$, were calculated (Fig. $5 B$ ).

Quantification of in vivo recovery interval $\left(t_{r}\right)$. Because any kinetic description of drug-induced CD is critically dependent on recovery from block during the interpulse recovery interval, epicardial MAP recordings were also examined in the presence of QUIN. In all experiments, QUIN prolonged epicardial MAPs and $A P D_{90}$ remained rate-dependent. Qualitatively, QUIN suppressed phase 1 repolarization resulting in an elimination of the spike and dome morphology of epicardial recordings and prolonged the plateau phase $\left(\mathrm{APD}_{50}\right)$ of the endocardial recordings. Fig. $6 A$ shows epicardial MAPs recorded near the left ventricular apex under steady-state conditions at ISI $=1.0 \mathrm{~s}$. Under control conditions, $\mathrm{APD}_{90}$ was $219 \mathrm{~ms}$ while with QUIN, $\mathrm{APD}_{90}$ prolonged to $262 \mathrm{~ms}$. In all experiments,

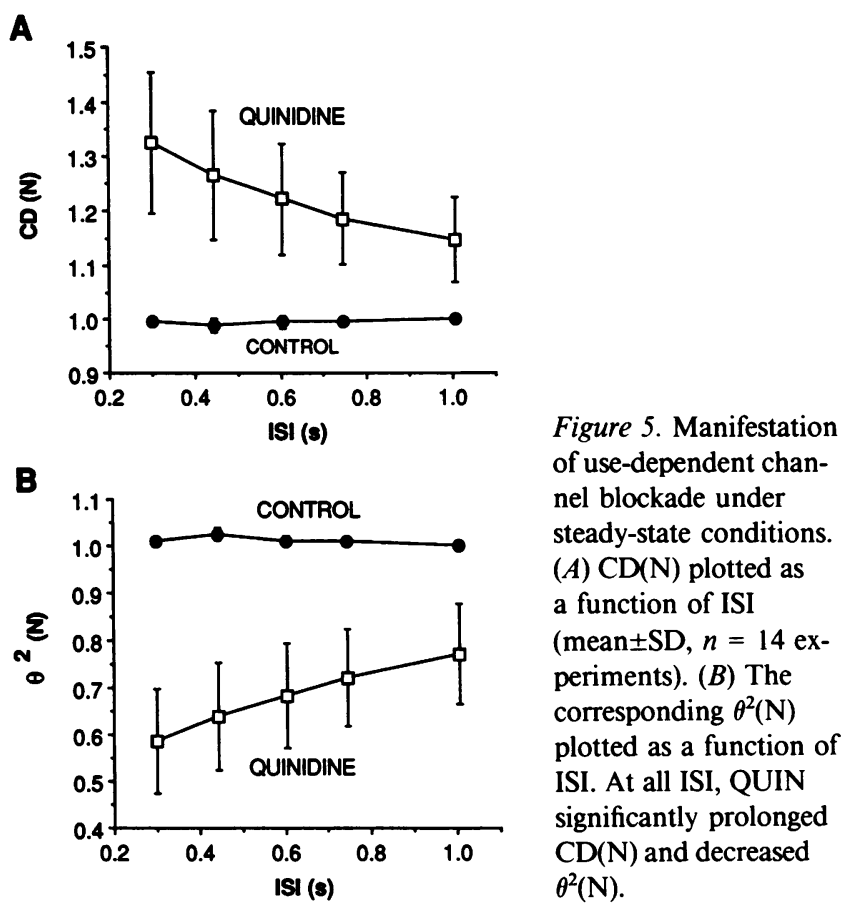

QUIN $A P D_{90}$ varied from $238 \pm 25$ at ISI $=1.0 \mathrm{~s}$ to $205 \pm 20$ at ISI $=0.3$, which is in contrast to the control $\mathrm{APD}_{90}$ of $210 \pm 18$ $\mathrm{ms}$ at ISI $=1.0$ and the value of $171 \pm 20 \mathrm{~ms}$ at ISI $=0.3$ (Fig. 6 $B$ ). At all ISIs, the prolongation of $\mathrm{APD}_{90}$ by QUIN was statisti-
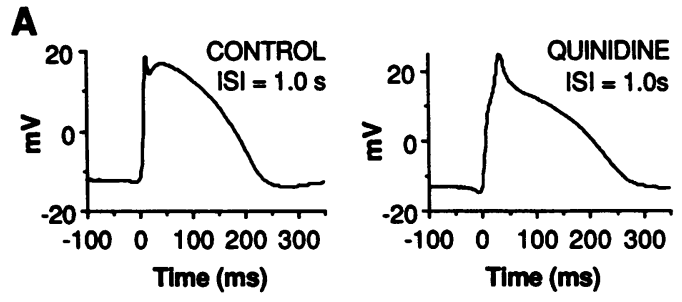

B

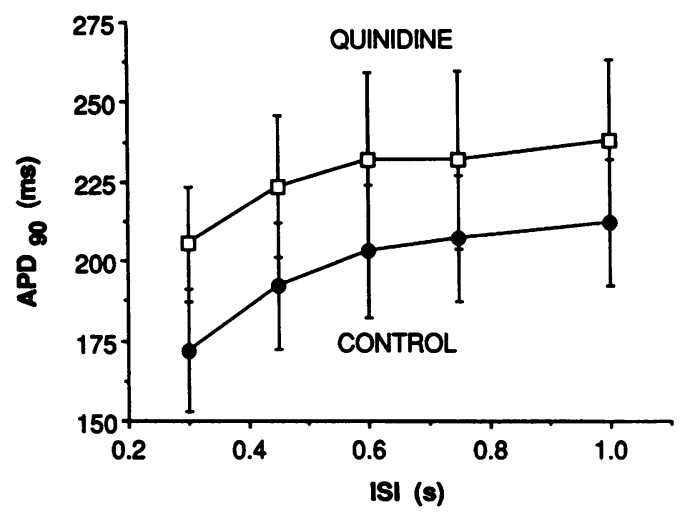

Figure 6. Effect of QUIN on epicardial monophasic action potential duration in vivo. $(A)$ Epicardial MAPs recorded under control conditions and in the presence of QUIN at ISI $=1.0 \mathrm{~s}$. Under control conditions, the action potential duration measured at $90 \%$ repolarization $\left(\mathrm{APD}_{90}\right)$ was $219 \mathrm{~ms}$. With QUIN, $\mathrm{APD}_{90}$ was prolonged to $262 \mathrm{~ms}$. (B) $\mathrm{APD}_{90}$ (mean $\pm \mathrm{SD}, n=14$ experiments) under control and experimental conditions plotted as a function of ISI. APD $_{90}$ 's were rate-dependent and decreased with decreasing ISI. At all ISI, QUIN significantly prolonged $\mathrm{APD}_{90}$. 
cally significant. From these data, the calculated recovery interval $\left(t_{\mathrm{r}}=\right.$ ISI $\left.-\mathrm{APD}_{90}\right)$ with QUIN was $762 \pm 25,518 \pm 28$, $368 \pm 27,227 \pm 22$, and $95 \pm 18 \mathrm{~ms}$ at ISIs of $1.0,0.75,0.6,0.45$, and $0.3 \mathrm{~s}$, respectively.

Analysis of the kinetics of QUIN-induced conduction delay under dynamic conditions. Using $\mathrm{CD}(\mathrm{N})$ and $\theta^{2}(\mathrm{~N})$ as reflections of QUIN binding, the time course of QUIN-induced block was examined during pulse train stimulation. As shown in Fig. $7 A$, repetitive stimulation produced beat-by-beat incremental $C D(N)$ with the greatest $C D(N)$ occurring at the shortest ISI. In agreement with the first formal prediction of the two-state model, $\mathrm{CD}(\mathrm{N})$ at each ISI rose with a mono-exponential time course to a new steady-state, as described by an apparent overall drug uptake rate, $\lambda^{*}$. In these studies, the estimation of $\lambda^{*}$ from $\theta^{2}(\mathrm{~N})$ was similar to those values derived from $\mathrm{CD}(\mathrm{N})$ (Fig. $7 \mathrm{~B}$ ). In the initial five experiments, the first 8-s acquisition was sufficient to characterize the time course of changing $C D(N)$, and gave similar results as seen in the last nine studies at comparable ISIs.

In each experiment, the relationship between $\lambda^{*}$ and $t_{\mathrm{r}}$ (ISIAPD) was linear, as shown in Fig. $8 \mathrm{~A}$, and as predicted by the expression $\lambda^{*}=\lambda_{\mathrm{a}} t_{\mathrm{a}}+\lambda_{\mathrm{r}} t_{\mathrm{r}}$. The $y$-intercept of the regression gave $\lambda_{\mathrm{a}}=121 \mathrm{~s}^{-1}$. From the slope of the relation, $\lambda_{\mathrm{r}}$ was found to be $0.44 \mathrm{~s}^{-1}$. The average values for all 14 experiments were $\lambda_{\mathrm{a}}$ $=147 \pm 67 \mathrm{~s}^{-1}$ and $\lambda_{\mathrm{r}}=0.61 \pm 0.26 \mathrm{~s}^{-1}$ (Table I). Similar results were derived using changes in $\theta^{2}(\mathrm{~N})$ as the reflection of QUIN block as shown in Fig. $8 B$ and Table I. The time course of QUIN recovery from block as estimated from the interpulse recovery during pulse train stimulation was compared with that determined by post-mature diastolic scanning over a 1.32.3-s diastolic interval in two experiments. Longer diastolic intervals were precluded by the emergence of spontaneous escape beats. The latter method yielded $\tau_{\mathrm{r}}=1.75$ and $1.77 \mathrm{~s}$ while $1 / \lambda_{\mathrm{r}}$ for those same experiments was 1.67 and $1.77 \mathrm{~s}$, respectively. Thus, the time course of recovery from QUIN block may be determined from the recovery which occurs during the interpulse recovery interval, $t_{\mathrm{r}}$.

Steady-state block, $r_{\mathrm{ss}}$, was also a linear function of the equilibrium expression $\gamma$, where $\gamma=\left(1-e^{-\lambda_{r} t_{r}}\right) /\left(1-e^{-\lambda^{*}}\right)$, as predicted. For the experiment illustrated in Fig. $9 A$, the activated state equilibrium block $a_{\infty}$, as derived y-intercept of the regression, was 0.50 and the slope of relation $\left(r_{\infty}-a_{\infty}\right)$, yielded $r_{\infty}$ $=0.08$, suggesting an $8 \%$ equilibrium or tonic block of resting $\mathrm{Na}^{+}$channels. The $a_{\infty}$ and $r_{\infty}$ values obtained in all 14 experiments from $\mathrm{CD}(\mathrm{N})$ were $a_{\infty}=0.41 \pm 0.16$, and $r_{\infty}=0.11 \pm 0.08$. Fig. $9 B$ shows a similar analysis performed using $\theta^{2}(\mathrm{~N})$.

Quantification of apparent fundamental rate constants. Using the experimental values obtained for $\lambda_{\mathrm{r}}, \lambda_{\mathrm{a}}, a_{\infty}, r_{\infty}$, and serum QUIN concentrations, the apparent rate constants $k_{\mathrm{a}}, l_{\mathrm{a}}$, $k_{\mathrm{r}}$, and $l_{\mathrm{r}}$ were calculated for all experiments (Table I). The
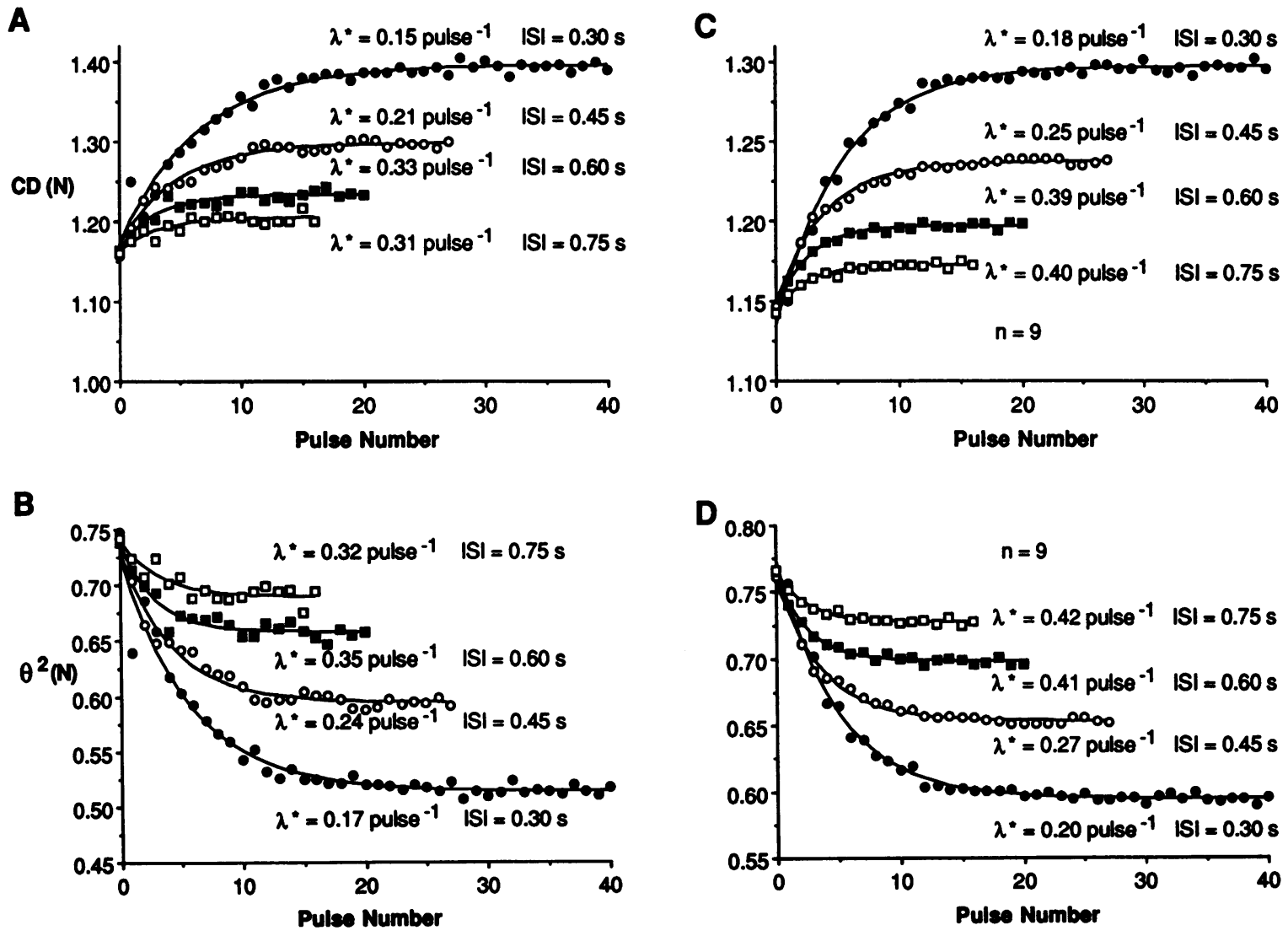

Figure 7. Dynamic manifestations of use-dependent channel block reflected by incremental conduction delay and declining $\theta^{2}(\mathrm{~N})$ at rapid heart rates in vivo. $(A)$ The incremental $C D(N)$ approaches new steady states with each pulse of 12 -s trains at ISIs of $0.3,0.45,0.6$, and $0.75 \mathrm{~s}$ from a baseline ISI of $1.0 \mathrm{~s}$. A nonlinear, least-squares technique was used to fit a single exponential curve to each set of experimental data. The resulting rate constant $\left(\lambda^{*}\right)$ represents the apparent drug uptake rate for each ISI. $(B)$ The corresponding decline in $\theta^{2}(\mathrm{~N})$ from the same data. $(C)$ The mean incremental $C D(N)$ obtained by averaging the $C D(N)$ data from each pulse of each train from 9 experiments. $(D)$ The mean declining $\theta^{2}(\mathrm{~N})$ obtained by similar averaging of $\theta^{2}(\mathrm{~N})$ data from nine experiments. 

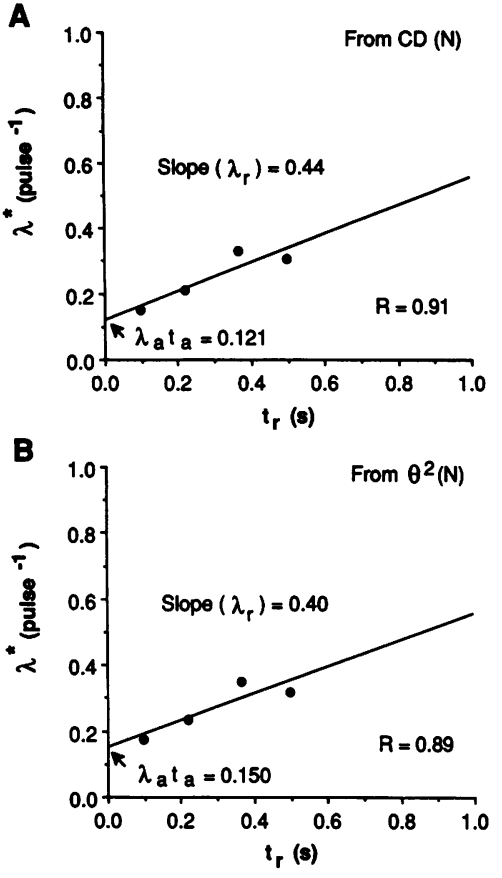

Figure 8. Estimation of state dependent drug uptake rates $\lambda_{\mathrm{r}}$ and $\lambda_{\mathrm{a}}$ in vivo from the relationship $\lambda^{*}=\lambda_{\mathrm{a}} t_{\mathrm{a}}+\lambda_{\mathrm{r}} t_{\mathrm{r}}$. (A) Linear regression of the overall drug uptake rate $\left(\lambda^{*}\right)$ determined from $\mathrm{CD}(\mathrm{N})$ as a function of recovery interval $\left(t_{\mathrm{r}}\right) . \lambda_{\mathrm{a}}$ was derived from the $y$-intercept where $t_{\mathrm{a}}=0.001$ s. $\lambda_{\mathrm{a}}$ is the drug uptake rate for the activated state and is related to the fundamental apparent binding rate constants by the expression $\lambda_{\mathrm{a}}=k_{\mathrm{a}} D+l_{\mathrm{a}}$. The slope of the line of regression is equal to $\lambda_{r}$, which is the drug uptake rate for the resting state and is related to the fundamental apparent bind-

ing rate constants by the expression $\lambda_{\mathrm{r}}=k_{\mathrm{r}} D+l_{\mathrm{r}}$. $(B)$ Linear regression of the overall drug uptake rate $\left(\lambda^{*}\right)$ determined from $\theta^{2}(\mathrm{~N})$ as a function of recovery interval $\left(t_{\mathrm{r}}\right)$.

apparent binding rate for activated channels derived from $\mathrm{CD}(\mathrm{N})\left(k_{\mathrm{a}}\right)$ was $7.1 \pm 3.5 \times 10^{6} \mathrm{M}^{-1} \mathrm{~s}^{-1}$. Calculated QUIN binding was $59 \mathrm{~s}^{-1}$. The unbinding rate from activated channels $\left(l_{\mathrm{a}}\right)$ was $81 \pm 51 \mathrm{~s}^{-1}$. This value was $>100$-fold greater than the apparent unbinding rate from resting channels $\left(l_{\mathrm{r}}\right)$ of $0.51 \pm 0.26$ $\mathrm{s}^{-1}$. The apparent binding rate to the resting channels $\left(k_{\mathrm{r}}\right)$ was $11.1 \pm 11.3 \times 10^{3} \mathrm{M}^{-1} \mathrm{~s}^{-1}$. Comparable calculations using $\theta^{2}(\mathrm{~N})$ as an in vivo marker of QUIN binding gave similar values of $l_{\mathrm{a}}$ and $l_{\mathrm{r}}$ and slightly greater values of $k_{\mathrm{a}}$ and $k_{\mathrm{r}}$ (Table I).

The effect of expected physiologic variation in beat-by-beat conduction on the rate constants so derived was also assessed by comparing results derived from individual preparations with rate constants derived from averaged $\mathrm{CD}(\mathrm{N})$ data from the final nine preparations. This averaging procedure incorpo-
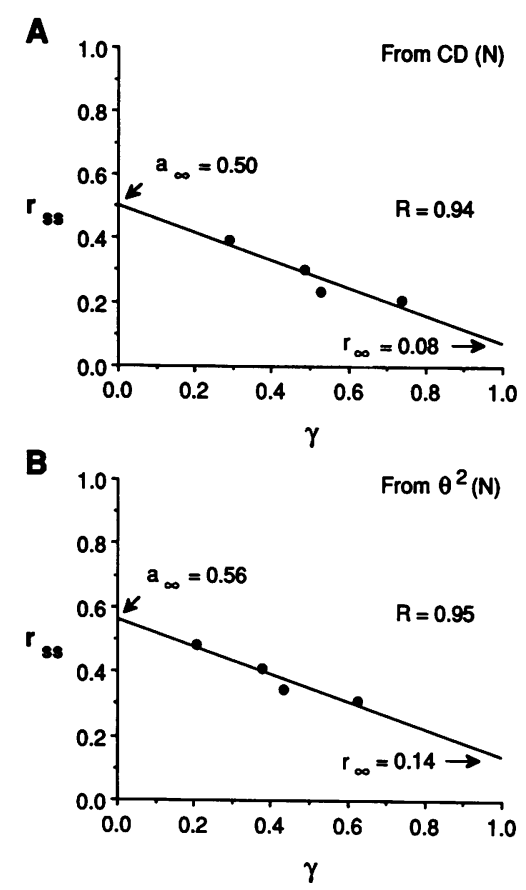

Figure 9. Estimation of equilibrium binding for the activated $\left(a_{\infty}\right)$ and resting $\left(r_{\infty}\right)$ states of $\mathrm{Na}^{+}$ channels in vivo. $(A) r_{\mathrm{ss}}$ [derived from $\mathrm{CD}(\mathrm{N})$ ] plotted as a function of the equilibrium expression $\gamma$, where $\gamma=(1$ $\left.-e^{-\left(\lambda_{r^{\prime}}\right)}\right) /\left(1-e^{-\lambda^{*}}\right)$. The $y$-intercept yields the value of equilibrium drug binding to the activated state, $a_{\infty}$, where $a_{\infty}=k_{\mathrm{a}} D /\left(k_{\mathrm{a}} D+l_{\mathrm{a}}\right)$. The slope of the regression is equal to the expression $a_{\infty}-r_{\infty}$ and is used to calculate equilibrium drug binding to the resting state, $r_{\infty}$, where $r_{\infty}=k_{\mathrm{r}} D /\left(k_{\mathrm{r}} D\right.$ $\left.+l_{\mathrm{r}}\right)$. (B) Similar results derived using $\theta^{2}(\mathrm{~N})$ as the marker of drug block.

rated $\mathrm{CD}(\mathrm{N})$ and $\theta^{2}(\mathrm{~N})$ for each pulse of each train at each given ISI (Fig. 7, $C$ and $D$ ) and yielded values of $\lambda^{*}$ which were similar to those seen in the individual experiments. In addition, the calculated drug uptake rates and rate constants were similar to those from the individual experiments, indicating little distortion of results from physiologic variations in $\mathrm{CD}$.

Effects of $A-V$ dissociation on quantification of QUIN-induced conduction delay in vivo. Careful examination of conduction times revealed that $\mathrm{CD}(\mathrm{N})$ changed as a function of the $\mathrm{AV}$ relationship. Observed $\mathrm{CD}(\mathrm{N})$ was typically slower with A-V synchrony. This effect is illustrated in Fig. $10 \mathrm{~A}$, which shows $\mathrm{CD}(\mathrm{N})$ plotted as a function of pulse number during a $12-\mathrm{s}$ pulse train at ISI $=0.3 \mathrm{~s}$. The spontaneous sinus rate was $0.59 \mathrm{~s}$, resulting in alternating $\mathrm{A}-\mathrm{V}$ synchrony and dyssynchrony. As emphasized in Fig. $10 B, C D(N)$ was $1.5-2 \%$ greater with, than without $\mathrm{A}-\mathrm{V}$ association. At the time indi-

Table I. Activated and Resting State QUIN Uptake Rates, Equilibrium Binding, and Apparent Rate Constants In Vivo

\begin{tabular}{|c|c|c|c|c|c|c|c|c|}
\hline & $\lambda_{2}$ & $\lambda_{r}$ & $a_{\infty}$ & $r_{\infty}$ & $k_{\mathrm{a}}$ & $l$. & $k_{\mathrm{T}}$ & $l_{\mathrm{r}}$ \\
\hline & $s^{-1}$ & $s^{-1}$ & & & $\times 10^{6} \mathrm{M}^{-1} \mathrm{~s}^{-1}$ & $s^{-1}$ & $\times 10^{3} \mathrm{M}^{-1} \mathrm{~s}^{-1}$ & $s^{-1}$ \\
\hline \multicolumn{9}{|c|}{$\begin{array}{l}\text { From incremental conduction delay* } \\
\qquad(n=14)\end{array}$} \\
\hline From $C D(N)$ & $147 \pm 67$ & $0.61 \pm 0.26$ & $0.41 \pm 0.16$ & $0.11 \pm 0.08$ & $7.1 \pm 3.5$ & $81 \pm 51$ & $11.1 \pm 11.3$ & $0.51 \pm 0.26$ \\
\hline From $\theta^{2}(\mathrm{~N})$ & $168 \pm 68$ & $0.59 \pm 0.27$ & $0.50 \pm 0.11$ & $0.18 \pm 0.12$ & $10.2 \pm 4.7$ & $84 \pm 42$ & $14.6 \pm 13.6$ & $0.48 \pm 0.22$ \\
\hline \multicolumn{9}{|c|}{$\begin{array}{l}\text { From decreasing conduction delay } \\
\qquad(n=13)\end{array}$} \\
\hline From $\mathrm{CD}(\mathrm{N})$ & $213 \pm 118$ & $0.64 \pm 0.31$ & $0.45 \pm 0.16$ & $0.08 \pm 0.07$ & $10.1 \pm 5.9$ & $130 \pm 89$ & $6.9 \pm 8.4$ & $0.55 \pm 0.31$ \\
\hline From $\theta^{2}(\mathrm{~N})$ & $226 \pm 108$ & $0.54 \pm 0.28$ & $0.56 \pm 0.18$ & $0.13 \pm 0.11$ & $13.4 \pm 7.1$ & $110 \pm 77$ & $9.1 \pm 9.6$ & $0.47 \pm 0.26$ \\
\hline
\end{tabular}

Abbreviations: $\lambda_{\mathrm{a}}$, activated state drug uptake rate; $\lambda_{\mathrm{r}}$, resting state drug uptake rate; $a_{\infty}$, activated state equilibrium binding; $r_{\infty}$, resting state equilibrium binding; $k_{\mathrm{a}}$, apparent activated state binding rate constant; $l_{\mathrm{a}}$, apparent activated state unbinding rate constant; $k_{\mathrm{r}}$, apparent resting state binding rate constant; $l_{\mathrm{r}}$, apparent resting state unbinding rate constant. Mean \pm SD. ${ }^{*}$ Determined from apparent drug uptake rates, $\lambda^{*}$. ${ }^{\ddagger}$ Determined from apparent drug decline rates, $R \lambda^{*}$. 


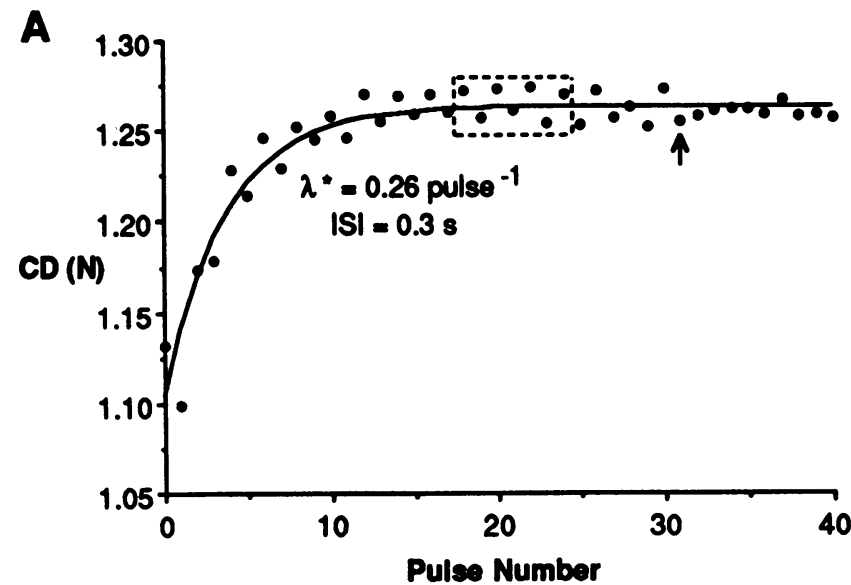

B

ECG
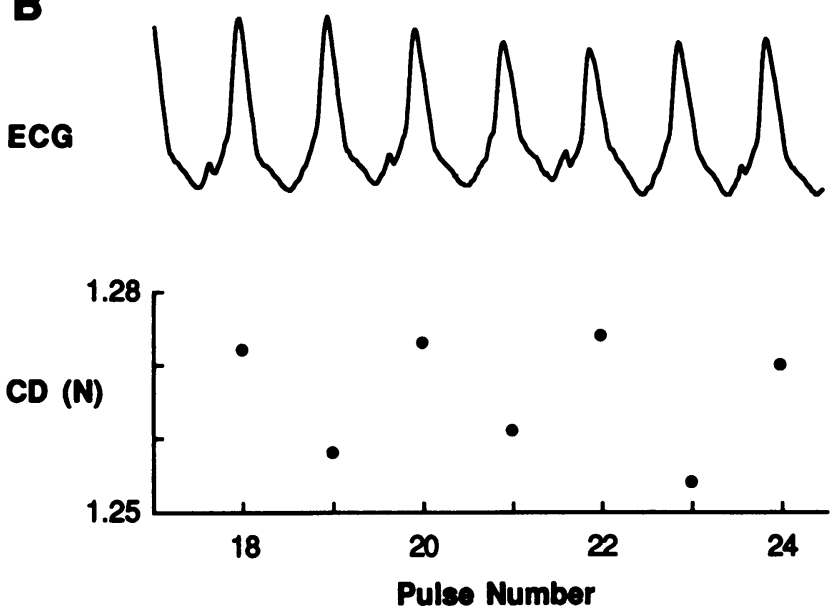

Figure 10. Effects of A-V synchrony on drug-induced conduction delay in vivo. $(A)$ Incremental $\mathrm{CD}(\mathrm{N})$ measured during a 12-s pulse train in the presence of QUIN at ISI $=0.3 \mathrm{~s}$. Note the cyclical alterations in $\mathrm{CD}(\mathrm{N})$ in the plateau phase of the response. During alterations, every other heart beat was preceded by a P-wave. At the time of an atrial premature contraction (arrow), the P-waves advanced into the $\mathrm{QRS}$ complexes so that no $\mathrm{QRS}$ was preceded by a P-wave. At that instant, the alternations in $\mathrm{CD}(\mathrm{N})$ disappeared. $(B)$ Expanded view of plateau region outlined in $A$ including surface ECG lead II.

cated by the arrow, a premature atrial contraction reset the sinus node, the $\mathrm{P}$-wave advanced into the $\mathrm{QRS}$, and the $\mathrm{CD}(\mathrm{N})$ alternations disappeared. In four experiments, sequential $\mathrm{A}-\mathrm{V}$ pacing restored A-V synchrony and abolished alternations in $\mathrm{CD}(\mathrm{N})$.

Decline of drug effect at decreasing heart rates. This quantitative analysis of QUIN kinetics including exponential fitting in vivo was facilitated by substantive changes in $\mathrm{CD}(\mathrm{N})$ produced over the range of pacing ISIs. Under conditions of increased baseline heart rates, as may exist in man, such an approach could be progressively limited by insufficient increments in $C D(N)$ with smaller step changes in pacing rates. Therefore, an alternative method for deriving apparent binding indices from the decline in drug effect accompanying stepping to a slower pacing rate was analyzed. In all experiments, $\mathrm{CD}(\mathrm{N})$ and $\theta^{2}(\mathrm{~N})$ were examined using a pulse protocol employing a 12 -s conditioning pulse train at ISI $=0.3 \mathrm{~s}$ to achieve high fractional block, followed by 12 -s pulse trains at ISI $=0.45,0.6$, 0.75 , and $1.0 \mathrm{~s}$, during which block diminished. As shown in
Fig. $11 A$, the change in $\mathrm{CD}(\mathrm{N})$ again followed a monoexponential time course to new steady-state levels, as use-dependent QUIN block declined with the decrease in pacing rates from ISI $=0.3$ to ISI $=0.45-1.0 \mathrm{~s}$. The experimental data were fit with a nonlinear, least squares method to define an overall drug decline rate $\left(R \lambda^{*}\right)$ from declining $\mathrm{CD}(\mathrm{N})$ at each ISI. In this and all other experiments, the largest $R \lambda^{*}$ was seen with the step in ISI from 0.3 to $1.0 \mathrm{~s}$ and $R \lambda^{*}$ decreased with decreasing ISI. As predicted by the two-state binding model, the drug uptake rates $\lambda^{*}$ and $R \lambda^{*}$ for a given ISI were similar despite differences in the direction from which that ISI was reached.

A plot of the relationship between $R \lambda^{*}$ [derived from $\mathrm{CD}(\mathrm{N})]$ and $t_{\mathrm{r}}$ is compared with the analogous plot of $\lambda^{*}$ and $t_{\mathrm{r}}$ from the same experiment in Fig. 11,C and $D$. The mean value of $\lambda_{\mathrm{r}}$ from declining $\mathrm{CD}(\mathrm{N})$ for all 13 studies was the same as that seen with $\lambda^{*}$ derivations from incremental $\mathrm{CD}(\mathrm{N})$ (Table I). $\lambda_{\mathrm{a}}$ derived from declining $\mathrm{CD}(\mathrm{N})$ tended to be greater than $\lambda_{\mathrm{a}}$ derived from incremental $\mathrm{CD}(\mathrm{N})$, however, although the difference was not statistically significant (Table I). Calculations of $\lambda_{\mathrm{a}}$ and $\lambda_{\mathrm{r}}$ from incremental $\theta^{2}(\mathrm{~N})$ were also similar to those obtained from declining $\mathrm{CD}(\mathrm{N})$. Plots of $r_{\mathrm{ss}}$ and $\gamma$ as derived from $R \lambda^{*}$ showed a similar linear relationship as seen with the abrupt increase in pacing rate and allowed calculation of activated and resting equilibrium block (Table I). $a_{\infty}$ and $r_{\infty}$ values from declining $\mathrm{CD}(\mathrm{N})$ were the same as those derived from incremental $\mathrm{CD}(\mathrm{N})$ (Table I). Calculations based on incremental $\theta^{2}(\mathrm{~N})$ data yielded modestly higher estimates of $a_{\infty}$ and $r_{\infty}$ (Table I).

Using the experimental values obtained for $\lambda_{\mathrm{r}}, \lambda_{\mathrm{a}}, a_{\infty}, r_{\infty}$ derived from declining $\mathrm{CD}(\mathrm{N})$, the apparent rate constants $k_{\mathrm{a}}$, $l_{\mathrm{a}}, k_{\mathrm{r}}$, and $l_{\mathrm{r}}$ were recalculated for all experiments (Table I) and compared with those obtained from $\lambda^{*}$ values from incremental $\mathrm{CD}(\mathrm{N})$ (Table I). Using $\mathrm{CD}(\mathrm{N})$ as a marker of drug binding, the apparent binding rate for activated channels $\left(k_{\mathrm{a}}\right)$ was $10.1 \pm 5.9 \mathrm{M}^{-1} \mathrm{~s}^{-1} \times 10^{6}$ and the apparent unbinding rate from activated channels $\left(l_{\mathrm{a}}\right)$ was $130 \pm 89 \mathrm{~s}^{-1}$. The apparent unbinding rate from the closed channels $\left(l_{\mathrm{r}}\right)$ was $0.55 \pm 0.31 \mathrm{~s}^{-1}$. Binding to the resting channels $\left(k_{\mathrm{r}}\right)$ was $6.9 \pm 8.4 \times 10^{3} \mathrm{M}^{-1} \mathrm{~s}^{-1}$. Similar values of apparent rate constants were obtained using $\theta^{2}(\mathrm{~N})$ as a marker of QUIN binding (Table I).

\section{Discussion}

This study demonstrates that in vivo $\mathrm{CD}$ as a manifestation of use-dependent channel block can be explicitly characterized in terms of apparent binding rate constants. By taking advantage of a high resolution technique which equally weights conduction times measured at each of 56 electrodes, this approach provides a sensitive quantitative description of drug-induced steady state and dynamic changes in conduction. We also found that under conditions of limited change in use-dependent block, this characterization could be enhanced by accounting for the effect of A-V synchrony on conduction times and by an analysis of the dynamics of declining block accompanying a decrease in pacing rate following high stimulation rate drug loading.

\section{Determination of apparent rate constants}

QUIN interaction with activated channels. Using these tools, a characterization of the acquisition of QUIN block during the activated state, $k_{\mathrm{a}}$, was available and found to be in agreement with previous estimates. We found that $k_{\mathrm{a}}=7.1 \times 10^{6} \mathrm{M}^{-1} \mathrm{~s}^{-1}$, which was similar to the value derived by Packer et al. (5) from 
A

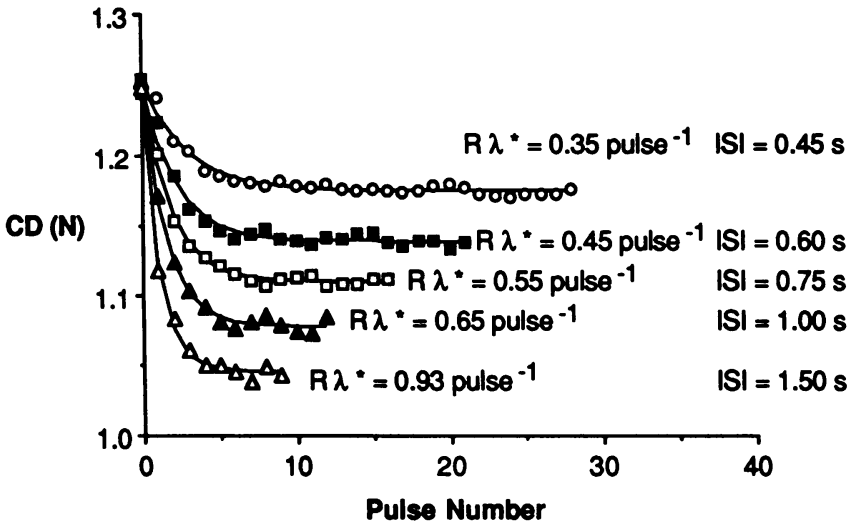

B

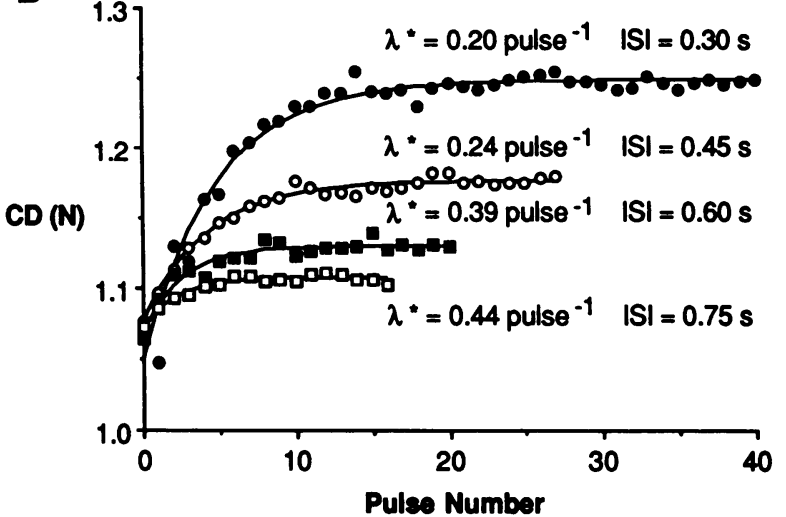

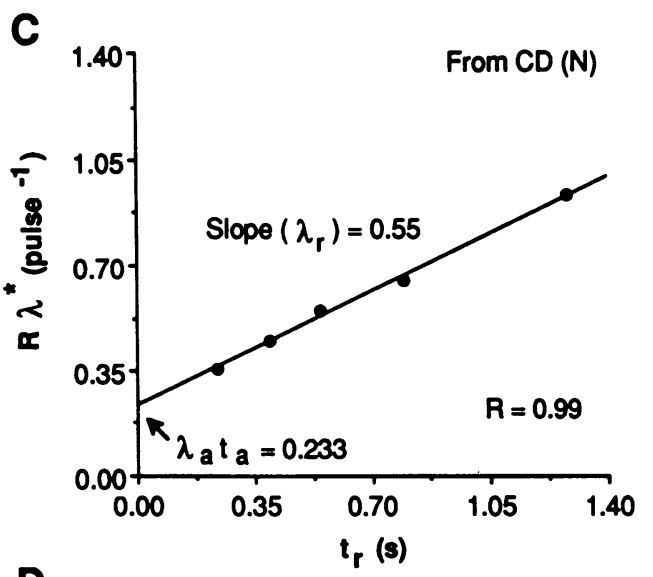

D

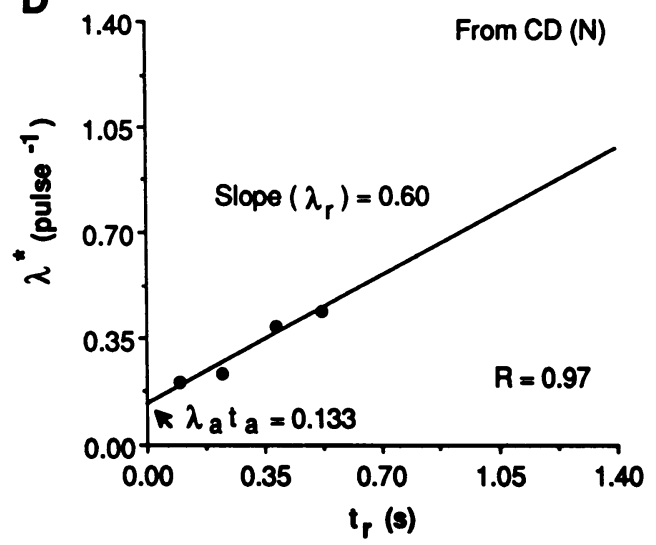

Figure 11. Comparison of use-dependent channel block derived from declining and incremental $\mathrm{CD}(\mathrm{N})$ in vivo. $(A)$ The stepwise decline in $\mathrm{CD}(\mathrm{N})$ observed at each pulse of a 12 -s train at ISIs of $0.45,0.6,0.75$, and 1.0 and $1.5 \mathrm{~s}$ after $12 \mathrm{~s}$ of pacing at an ISI of $0.3 \mathrm{~s}$. A nonlinear, least-squares technique was used to fit a single exponential curve to each experimental data set. The resulting rate constants represent the apparent drug decline rates $\left(R \lambda^{*}\right)$ for pulse train stimulation at each ISI. $(B)$ Incremental $C D(N)$ observed with each pulse of a 12-s train at ISIs of $0.3,0.45,0.6$, and $0.75 \mathrm{~s}$ from the baseline ISI of $1.0 \mathrm{~s}$. The accompanying rate constants represent the apparent drug uptake rate $\left(\lambda^{*}\right)$ for pulse train stimulation at each ISI. (C) Linear regression of the overall drug decline rate $\left(R \lambda^{*}\right)$ determined from $\mathrm{CD}(\mathrm{N})$ as a function of recovery interval $\left(t_{\mathrm{r}}\right)$. Again, the $y$-intercept is $\lambda_{\mathrm{a}} t_{\mathrm{a}}$, where $t_{\mathrm{a}}=0.001 \mathrm{~s}$ and $\lambda_{\mathrm{a}}$ is the drug uptake rate for the activated state. The slope of the regression line is $\lambda_{\mathrm{r}}$, the drug uptake rate for the resting state. $(D) \lambda^{*}$ plotted as a function of $t_{\mathrm{r}}$.

$\mathrm{CD}$ in isolated canine Purkinje fibers. Hondeghem and Katzung (2) obtained a value of $k_{\mathrm{a}}$ that was larger $\left(26.0 \times 10^{6} \mathrm{M}^{-1}\right.$ $\mathrm{s}^{-1}$ ), however, which could be accounted for by the shorter 0.3-ms activated state uptake time applied in the latter calculations of $k_{\mathrm{a}}$. Our estimated unbinding rate from the activated channel $l_{\mathrm{a}}=81 \mathrm{~s}^{-1}$, was also on the same order as those derived previously from Purkinje fibers (5) and that predicted by Hondeghem and Katzung (2).

QUIN interaction with the resting channel. In these studies, $9-16 \%$ tonic block was present. Although several quantitative analyses have assumed that tonic block was zero $(13,23,28)$, this finding is compatible with previous experimental observations. Under low-temperature voltage-clamp conditions, Lee et al. (29) observed $60 \%$ tonic block in the presence of $20 \mu \mathrm{M}$ QUIN. More recently, Snyders and Hondeghem (24) found that tonic block in vitro ranged from undetectable levels to $11-20 \%$ as the QUIN concentration was increased from 5 to 50 $\mu \mathrm{M}$. $4 \%$ tonic block in the presence of QUIN has also been described in simple propagating systems (5).

Other potential contributors to higher "apparent" tonic block in vivo include $(a)$ preparation rundown during the experiment and $(b)$ the possibility of ventricular dilatation associated with volume loading producing longer apparent conduc- tion times. Rundown of the preparation, however, was not observed in control animals in which repeat assessment of conduction times were made over a prolonged period equivalent to that required for the complete QUIN studies. The latter explanation was also unlikely since repeat control data acquired after a 35-min saline infusion to mimic QUIN loading and maintenance infusions produced no increase in normalized CD. Furthermore, no appreciable increases in conduction time accompanied ventricular dilatation related to maximal pauses between pacing trains in the presence of QUIN. Greater tonic block could also reflect the presence of a second, slower (>35 s) component of recovery from QUIN block in vitro as suggested previously (24), although such slow components have not been described at physiologic temperatures. Based on this $r_{\infty}$, the forward binding rate in resting channels, $k_{\mathrm{r}}$, was $9.1 \pm 14.6 \times 10^{3} \mathrm{M}^{-1} \mathrm{~s}^{-1}$, which was higher than values previously observed in in vitro studies with lower tonic block.

In contrast, the unbinding rates observed in this study were less than those previously described in vitro. We found that $\tau_{\mathrm{r}}$ $=1.9 \mathrm{~s}$, which was faster than the 4-6 s values observed in vitro $(5,23,30)$. Consistent with these observations, a new steadystate level of block during pacing at 0.3-1.0-s interstimulus intervals was reached within $6 \mathrm{~s}$. In addition, our estimates of $\tau_{\mathrm{r}}$ 
for QUIN extracted from the interpulse recovery interval were in agreement with the $\tau_{\mathrm{r}}$ values obtained from limited post-mature impulse diastolic scanning that were also faster than previous in vitro values. These findings, however, are consistent with previous studies demonstrating more rapid recovery in vivo than predicted from in vitro data. Nattel and Jing (14) found a $\tau_{\mathrm{r}}=1.6 \mathrm{~s}$ for procainamide, which was $\sim 50 \%$ faster than previously observed in vitro values. Furthermore, Bajaj et al. (13) estimated a QUIN $\tau_{\mathrm{r}}=2.2 \pm 6.6 \mathrm{~s}$ from in vivo canine studies in which recovery kinetics were examined with postmature stimulus scanning of the diastolic interval. Our estimates of $\tau_{\mathbf{r}}$ for QUIN extracted from the interpulse recovery interval similarly were in agreement with values obtained from similar post-mature impulse diastolic scanning. The reason for faster in vivo recovery than seen in vitro is unclear. Since these preparations were denervated, it is unlikely that neural sympathetic input contributed to this more rapid recovery. In contrast, it is possible that circulating catecholamines modulate QUIN effect in vivo (31-33) by accelerating recovery from drug block.

\section{Elucidation of mechanism of use-dependent block}

Comparisons of these state-dependent apparent rate constants yield insight into the mechanisms of use-dependent block in vivo. The large value of $k_{\mathrm{a}}\left(7.1 \pm 3.5 \times 10^{6} \mathrm{M}^{-1} \mathrm{~s}^{-1}\right)$ relative to $k_{\mathrm{r}}$ $\left(6.9 \pm 8.4 \times 10^{3}\right)$ confirms the assumption from in vitro studies that QUIN is largely an open state blocker in vivo (22-24). The open state blocking property of QUIN is consistent with the actions of other $\mathrm{Na}^{+}$channel-blocking agents which are in a charged form at physiologic $\mathrm{pH}$ (34) and presumes increased accessibility of the drug binding site via a hydrophilic pathway. At resting membrane potentials where the closed form of the channel predominates, drug binding is reduced, perhaps by channel protein conformational changes which stearically hinder hydrophilic access of drug to the channel. Interestingly, a comparison of $l_{\mathrm{a}}$ and $l_{\mathrm{r}}$ also suggested the presence of drug trapping under resting conditions. This is consistent with an impeded dissociation of QUIN from resting channels. In contrast, the comparatively large value for $l_{\mathrm{a}}=0.85 \mathrm{~s}^{-1}$ is compatible with unimpeded eggression of the charged QUIN moiety from the activated channel via a hydrophilic pathway. In light of these molecular interpretations of QUIN binding, it should be emphasized that the apparent action of QUIN under the conditions of our study is quite simple and may be adequately described by the two-state (four rate constant) model which we have employed. This analytical approach is a straightforward means of quantifying drug-channel interaction with a minimal number of assumptions and is an algebraic derivation based on a two-state binding model. As such, this analysis does not require a "guarded" $(1)$ or "modulated" $(2,3)$ receptor. Subsequently, from these apparent binding rate constants, either interpretation can be invoked to speculate on the underlying binding mechanism.

\section{Effect of $A$-V synchrony}

A-V synchrony was found to increase detectable epicardial conduction times in the presence of QUIN. To our knowledge, this phenomenon has not been previously described. While the exact mechanism by which ventricular activation is slowed by atrial preload is unknown, the possibility of a neurally mediated or reflex process is excluded in our denervated preparation. Alternatively, the rapid time course of the response
( $<100 \mathrm{~ms}$ ) suggests a physical or stretch activated mechanism. Specifically, the increase in ventricular size increases the effective electrical distance, via sarcolemmal unfolding, between two recording sites (35) thus increasing conduction times. In support of this are the observations of Calkins et al. (36), who found a small but significant increase in left ventricular activation time in response to volume loading of the isolated canine heart. In contrast to previous investigations where ventricular stretch elicited diastolic depolarization and automaticity (3739), however, we saw no additional ectopy with A-V synchrony. We speculate that this difference is a matter of degree: with mild ventricular enlargement conduction slows, while greater stretch elicits depolarization and ectopy.

Any such changes in conduction time resulting from variability in the A-V relationship has the potential of introducing error into the quantification of drug-induced CD particularly in the setting of small incremental changes in conduction. In this study, however, the effect related to A-V synchrony was insufficient to obscure the observed changes in $\mathrm{CD}$ resulting from changes in stimulus rate from 1.0 to $0.45-0.3 \mathrm{~s}$. The apparent binding rate constants derived from exponential fits of averaged $C D$ in nine experiments were the same as those obtained by analysis of individual experiments provides further validation of our results. Nevertheless, sequential A-V pacing may be helpful in those instances where dynamic changes in $\mathrm{CD}$ are small, as may be seen with low drug concentrations or where the step change in ISI and, therefore, incremental CD, is limited by rapid baseline heart rates.

\section{Binding characterization from declining conduction delay}

Our finding that drug action can be comparably characterized from either increasing or decreasing $\mathrm{CD}$ or analogous changes in $\theta^{2}(\mathrm{~N})$ holds significant theoretical and clinical implications. This observation supports the use of a two-state model to describe in vivo QUIN effect over a broad range of physiologic heart rates. The relationship $\lambda^{*}=\lambda_{\mathrm{a}} t_{\mathrm{a}}+\lambda_{\mathrm{r}} t_{\mathrm{r}}$ predicts that overall drug binding rates are dependent on the recovery interval, $t_{\mathrm{r}}$, but independent of the direction from which the steady-state block is approached. This theoretical equivalence of $\lambda^{*}$ determined from declining $\theta^{2}(\mathrm{~N})$ and $R \lambda^{*}$ derived from incremental $\theta^{2}(\mathrm{~N})$ is demonstrated in Fig. $12 A$, which shows computer simulations of the time course of $\theta^{2}(\mathrm{~N})$ approach to steady state at ISI $=0.6 \mathrm{~s}$ after either a step decrease in ISI from 0.3 to $0.6 \mathrm{~s}$ or a step increase in ISI from 1.0 to $0.6 \mathrm{~s}$. Here, the drug uptake $\left(\lambda^{*}\right)$ and drug decline $\left(R \lambda^{*}\right)$ rates are identical. In support of the theoretical equivalence of $\lambda^{*}$ and $R \lambda^{*}$, averaged $\lambda^{*}$ and $R \lambda^{*}$ data at ISI $=0.6 \mathrm{~s}$ derived from the final nine experiments were nearly identical (Fig. $12 \mathrm{~B}$ ). In addition, both $\lambda^{*}$ and $R \lambda^{*}$ are linear functions of $t_{\mathrm{r}}$ and yield identical expressions of interpulse drug recovery, $\lambda_{\mathrm{r}}$. While a trend towards a larger $\lambda_{\mathrm{a}}$ was observed with derivations from incremental $\theta^{2}(\mathrm{~N}), a_{\infty}, r_{\infty}$, and the fundamental rate constants derived from both shifts in $\theta^{2}(\mathrm{~N})$ were comparable. The use of declining $\mathrm{CD}$ [or incremen$\left.\operatorname{tal} \theta^{2}(\mathrm{~N})\right]$ in the examination of QUIN kinetics may provide a more robust description of the time course of block occurring at pacing cycle lengths near the baseline heart rates, where changes in incremental $C D$ with an increase in pacing rate from baseline produce drug-induced changes in conduction which are too small to resolve. In contrast, incremental CD may provide a better measure of the time course of block under conditions of large increases in pacing rate where the apparent drug-induced change in conduction is greater. The similarity in 


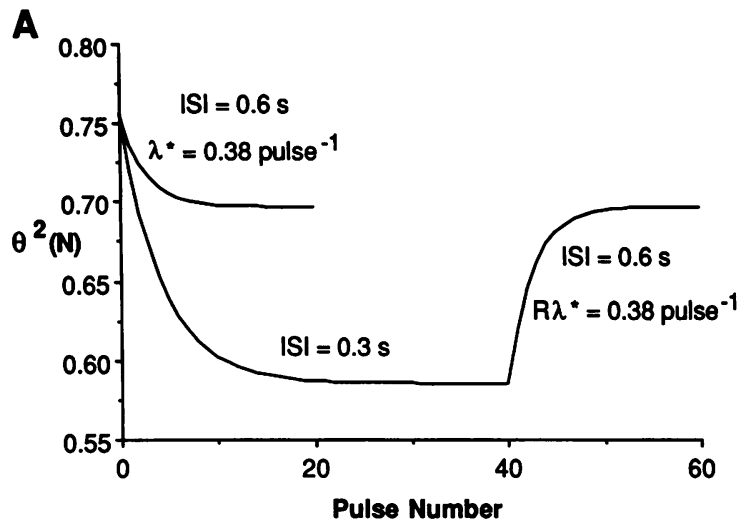

B

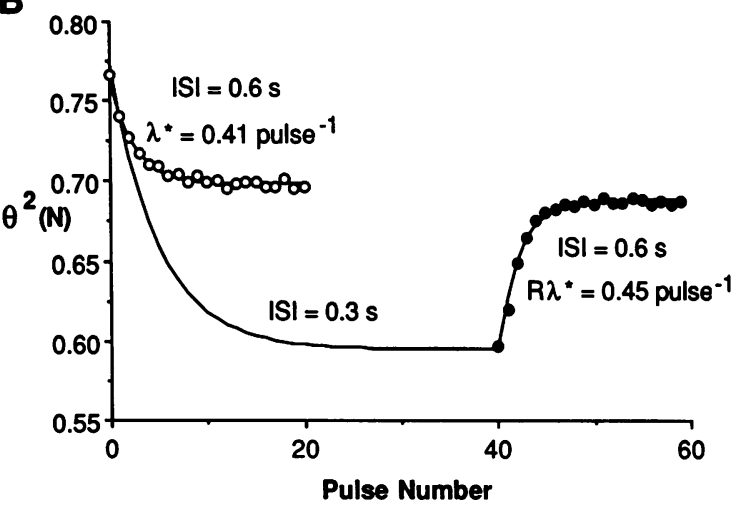

Figure 12. Theoretical and experimental validation of the equivalency of $\lambda^{*}$ and $R \lambda^{*}$ for estimation of apparent binding rates in vivo. $(A)$ Computer-generated simulations of predicted dynamic changes in $\theta^{2}(\mathrm{~N})$. In response to a step change in ISI from 1.0 to $0.6 \mathrm{~s}$ (upper trace), the predicted $\theta^{2}(\mathrm{~N})$ declines monoexponentially with a drug uptake rate of $\lambda^{*}=0.38$ pulse $^{-1}$. In response to sequential step changes in ISI from 1.0 to 0.3 and then to $0.6 \mathrm{~s}$ (lower trace), the predicted $\theta^{2}(\mathrm{~N})$ declines monoexponentially and then, at ISI $=0.6 \mathrm{~s}$ increases monoexponentially with a drug decline rate of $R \lambda^{*}=0.38$ pulse $^{-1}$. The two-state model predicts that $\lambda^{*}$ and $R \lambda^{*}$ at ISI $=0.6 \mathrm{~s}$ are equivalent. (B) Averaged experimentally observed $(n=9)$ incremental $\theta^{2}(\mathrm{~N})$ and declining $\theta^{2}(\mathrm{~N})$ during pulse trains at ISI $=0.6 \mathrm{~s}$. In response to a step change in ISI from 1.0 to $0.6 \mathrm{~s}(\mathrm{O}), \theta^{2}(\mathrm{~N})$ declined monoexponentially with a drug uptake rate of $\lambda^{*}=0.41$ pulse $^{-1}$. In response to sequential step changes in ISI from 1.0 to 0.3 and then to $0.6 \mathrm{~s}(\bullet), \theta^{2}(\mathrm{~N})$ declined monoexponentially and then, at ISI $=0.6$ $s$ increased monoexponentially with a drug decline rate of $R \lambda^{*}=0.45$ pulse $^{-1}$. A nonlinear, least-squares technique was used to fit single exponential curves to the two sets of experimental data.

binding rates derived from both incremental and decremental CD also suggests that $\lambda^{*}$ and $R \lambda^{*}$ data might be combined where necessary to explore the kinetics of drug binding under a highly restricted range of pacing rates.

MAPS

Our observations from epicardial MAPs under control conditions and with QUIN were in agreement with previous in vivo (40) and in situ (41) investigations. Control epicardial APD 90 's showed significant rate dependence as previously described $(40,42,43)$. With QUIN, APD $_{90}$ 's remained rate-dependent but were prolonged at all ISIs by $20-25 \mathrm{~ms}$, similar to that observed by Imaizumi and Giles (44) in vitro, Franz and Costard (41) in the intact canine heart, and Nademanee et al. (42) in humans. Under control conditions, epicardial MAPs exhib- ited a pronounced phase 1 repolarization ("spike and dome" morphology), consistent with that due to transient outward currents (45-47). Furthermore, with rapid pacing, this component was attenuated as previously described $(40,45,47)$. QUIN also suppressed phase 1 repolarization with a resultant loss of the spike and dome morphology of epicardial MAPs. This observation is compatible with the demonstration of Imaizumi and Giles (44) that QUIN blocks transient outward current.

\section{Limitations}

The quantitative tools employed in this analysis require that epicardial activation sequences remain constant during control and drug states at each ISI within each experiment. Changes in the sequence of ventricular activation, if present, could be inappropriately interpreted as drug-induced $C D$ and therefore confound binding rate determination. That this was not the case in this study was insured by two factors: $(a)$ the monoexponential time course of $\mathrm{CD}$ over each stimulation train was continuous; and $(b)$ the plots of conduction times for all electrodes in the presence of QUIN as a function of similar values from a control heartbeat for every heartbeat analyzed were linear. The preservation of activation sequence in the presence of procainamide, indicated by precise linearity in the relationship between conduction times at each epicardial electrode site normalized to the longest conduction time for a given impulse during drug states similarly normalized to control values, has been similarly demonstrated by Nattel et al. (14). It seems reasonable to conclude that activation sequence was highly preserved during the dynamic conditions of pulse-train stimulation used to quantify incremental CD.

The estimation of $t_{\mathrm{r}}$ was typically based on a single site determination of epicardial action potential duration. Although regional variations in $\mathrm{APD}_{90}$ occur, we and others (40, 43) have found that differences between epicardial and endocardial ventricular $\mathrm{APD}_{90}$ 's are $<30 \mathrm{~ms}$ at physiologic heart rates. Given the magnitude of ISIs used, this level of variation in $\mathrm{APD}_{90}$ would not alter significantly the relation between $\lambda^{*}$ and $t_{\mathrm{r}}$. Furthermore, the change of APD ${ }_{90}$ over the course of a pulse train was insufficient to confound the use of a single $\mathrm{APD}_{90}$ value in $t_{\mathrm{r}}$ calculations at a given ISI.

This characterization of QUIN action presumes that changes in measures of conduction due to drug are a reflection of $\mathrm{Na}^{+}$channel block. This assumption is supported by previous in vitro analyses of use-dependent drug effects showing that $\theta^{2}$ and $V_{\max }$ in the presence of drug remain linearly related $(4,5)$ in the absence of appreciable membrane depolarization (48). Additional support is provided by the derivation of nearly identical binding rates describing in vitro QUIN (5) and lidocaine (49) block obtained from both $V_{\max }$ and $\theta^{2}$. Nattel (6) also demonstrated agreement between moderate degrees of lidocaine-induced $C D$ and $V_{\max }$ suppression in vitro. The relationship between $V_{\max }$ and $g_{\mathrm{Na}}$, also appears to be more linear at physiologic temperatures. Johns et al. (50) showed that the correlation between $V_{\max }$ and $l_{\mathrm{Na}}$ measures of block induced by O-demethyl encainide improved with increasing temperature to $25^{\circ} \mathrm{C}$. An improvement in the agreement between $g_{\mathrm{Na}}$ and $V_{\max }$ with increasing temperature from $7^{\circ}$ to $27^{\circ} \mathrm{C}$ has also been documented by others (51). Thus the analysis of use-dependent $\mathrm{Na}^{+}$channel block from measures of conduction seems justified. Even with distortion of the relationship between $\theta^{2}$ and $g_{\mathrm{Na}}(52)$, these binding rates still provide an index 
of use dependence which will be useful in examining drug effect under a variety of conditions.

\section{Clinical relevance}

The data presented herein demonstrate that apparent binding rates describing antiarrhythmic drug action over a range of physiologic heart rates can be derived from in vivo use-dependent $C D$. Our results suggest that similar methods could be applied to analyze use-dependent drug effects and validate apparent binding rate determination in man. The use of multiple intracardiac or epicardial bipolar recordings in conjunction with simple pacing protocols may allow a sensitive, high-resolution analysis of drug-induced $C D$ exceeding that which could be obtained from prolongation of the surface QRS. The apparent binding rates for various antiarrhythmic drugs to human myocardium obtained under clinically relevant conditions may have implications regarding action, efficacy, and proarrhythmia. For example, from these rate constants, it should be possible to predict physiologic conduction delay generated by a given drug concentration at a given pacing rate. Furthermore, this information can be incorporated into more complex models that also accommodate changes in refractoriness and the contribution of passive properties to more completely describe drug action. This analysis may also disclose tissue-dependent regional variations in drug-induced $C D$ related to underlying abnormalities such as the presence of myocardial infarction. Given the general relationship between time constants of recovery from drug block and proarrhythmia potential (53), the propensity for a drug-related arrhythmia aggravation may also be evaluated. Clinical studies could reveal interindividual variations in apparent binding rates to help explain why certain patients are more sensitive to a given antiarrhythmic agent than others. It is also possible that such physiologic data could eventually be analyzed in conjunction with molecular genetic analysis of ion channel drug binding sites to identify potential mechanisms for abnormal or toxic responses to therapy in humans. Most importantly, binding rates derived from alterations in drug-induced $C D$ observed at the level of the intact heart could be analyzed under conditions of acidosis, hypoxia, ischemia, or hyperadrenergic states such as might be seen with congestive heart failure or acute myocardial infarction. This might enhance the ability to identify those drugs which exhibit greater effect or less toxicity with such changes in a given patient's clinical status. Although largely speculative at this juncture, the potential implications for antiarrhythmic drug binding rate determination in humans by the in vivo methods we have applied are significant and warrant further clinical investigation.

\section{Acknowledgments}

The authors thank Ms. Monica Zeien for her assistance in manuscript preparation.

This study was supported in part by a grant-in-aid from the $\mathrm{Na}$ tional American Heart Association (Dr. Packer).

\section{References}

1. Starmer, C. F., A. O. Grant, and H. C. Strauss. 1984. Mechanisms of use-dependent block of sodium channels in excitable membranes by local anesthetics. Biophys. J. 46:15-27.

2. Hondeghem, L. M., and B. G. Katzung. 1977. Time- and voltage-dependent interactions of antiarrhythmic drugs with cardiac sodium channels. Bio chim. Biophys. Acta. 472:373-398.
3. Hille, B. 1977. Local anesthetics: hydrophilic and hydrophobic pathways for the drug receptor reaction. J. Gen. Physiol. 69:497-515.

4. Buchanan, J. W., T. Sarto, and L. S. Gettes. 1985. The effects of antiarrhythmic drugs, stimulation frequency, and potassium-induced resting membrane potential changes on conduction velocity and $d V / d t_{\max }$ in guinea pig myocardium. Circ. Res. 56:696-703.

5. Packer, D. L., A. O. Grant, H. C. Strauss, and C. F. Starmer. 1989. Characterization of concentration- and use-dependent effects of quinidine from conduction delay and declining conduction velocity in canine Purkinje fibers. $J$. Clin. Invest. 83:2109-2119.

6. Nattel, S. 1987. The relationship between frequency-dependence of local anesthetic drug effects on $V_{\max }$ and conduction in canine cardiac Purkinje fibers. J. Pharmacol. Exp. Ther. 241:282-288.

7. Gettes, L. S., B. Surawicz, and J. C. Shiue. 1962. Effect of high K, low K and QUIN on QRS duration and ventricular action potential. Am. J. Physiol. 203:1135-1140.

8. Wallace, A. G., R. E. Cline, W. C. Sealy, W. G. Young, Jr., and W. G. Troyer. 1966. Electrophysiologic effects of QUIN: studies using chronically implanted electrodes in awake dogs with and without cardiac denervation. Circ. Res. 19:960-969.

9. Gang, E. S., T. A. Denton, D. S. Oseran, W. J. Mandel, and T. Peter. 1985. Rate-dependent effects of procainamide on His-Purkinje conduction in man. Am. J. Cardiol. 55:1525-1529.

10. Morady, F., L. DiCarlo, J. Boerman, and B. Crevey. 1985. Rate-dependent effects of intravenous lidocaine, procainamide and amiodarone on intraventricular conduction. J. Am. Coll. Cardiol. 6:179-185.

11. Ranger, S., M. Talajic, R. Lemery, D. Roy, and S. Nattel. 1989. Amplification of flecainide-induced ventricular conduction slowing by exercise: a potentially significant clinical consequence of use-dependent sodium channel blockade. Circulation. 79:1000-1006.

12. Davis, J., T. Matsubara, M. M. Scheinman, B. Katzung, and L. H. Hondeghem. 1986. Use-dependent effects of lidocaine on conduction in canine myocardium: application of the modulated receptor hypothesis in vivo. Circulation. 74:205-214.

13. Bajaj, A. K., H. A. Kopelman, J. P. Wikswo, F. Cassidy, R. L. Woosley, and D. R. Roden. 1987. Frequency- and orientation-dependent effects of mexilitine and quinidine on conduction in the intact dog heart. Circulation. 75:10651073.

14. Nattel, S., and W. Jing. 1989. Rate-dependent changes in intraventricular conduction produced by procainamide in anesthetized dogs: a quantitative analysis based on the relation between phase 0 inward current and conduction velocity. Circ. Res. 65:1485-1498.

15. Villemaire, C., P. Savard, M. Talajic, and S. Nattel. 1992. A quantitative analysis of use-dependent ventricular slowing by procainamide in anesthetized dogs. Circulation. 85:2255-2266.

16. Starmer, C. F., and A. O. Grant. 1985. Phasic ion channel blockade: a kinetic model and parameter estimation procedure. Mol. Pharmacol. 38:348356.

17. Starmer, C. F., D. L. Packer, and A. O. Grant. 1986. Ligand binding to transiently accessible sites: mechanisms for varying apparent binding rates. $J$. Theor. Biol. 124:335-341.

18. Steiner, C., and A. T. W. Kovalik. 1968. A simple technique for production of chronic complete heart block in dogs. J. Appl. Physiol. 25:631-632.

19. Lindmar, R., K. Loffelholz, and W. Weide. 1979. Inhibition by pentobarbital of the acetylcholine release from the postganglionic parasympathetic neuron of the heart. J. Pharmacol. Exp. Ther. 210:166-173.

20. Gillaim, F. R., C. F. Starmer, and A. O. Grant. 1989. Blockade of rabbit atrial sodium channels by lidocaine: characterization of continuous and frequency-dependent blocking. Circ. Res. 65:723-739.

21. Kojima, M., and T. Ban. 1988. Nicorandil shortens action potential duration and antagonizes the reduction of $V_{\max }$ by lidocaine but not disopyramide in guinea pig papillary muscles. Naunyn-Schmiedebergs Arch. Pharmakol. 337:203-212.

22. Colatsky, T. J. 1983. Quinidine block of cardiac $\mathrm{Na}^{+}$channels is rate- and voltage-dependent. Biophys. J. 37:343a. (Abstr.)

23. Weld, F. M., J. Coromilas, J. N. Rottman, and J. T. Bigger, Jr. 1982. Mechanisms of QUIN-induced depression of maximum upstroke velocity in ovine cardiac Purkinje fibers. Circ. Res. 50:210-217.

24. Snyders, D. J., and L. M. Hondeghem. 1990. Effects of quinidine on the sodium current of guinea pig ventricular myocytes: evidence for a drug-associated resting state with altered kinetics. Circ. Res. 66:565-579.

25. Anno, T., and L. M. Hondeghem. 1990. Interactions of flecainide with guinea pig cardiac $\mathrm{Na}^{+}$channels: importance of activation unblocking to the voltage dependence of recovery. Circ. Res. 66:789-803.

26. Grant, A. O., M. A. Dietz, F. R. Gilliam III, and C. F. Starmer. 1989. Blockade of cardiac sodium channels by lidocaine: single channel analysis. Circ. Res. 65:1247-1262.

27. Grant, A. O., and C. F. Starmer. 1987. Mechanism of closure of sodium channels in rabbit ventricular myocytes: single channel analysis. Circ. Res 60:897-913. 
28. Heistracher, P. 1971. Mechanism of action of antifibrillatory drugs. Naunyn-Schmiedeberg's Arch. Pharmakol. 269:199-212.

29. Lee, K. S., J. R. Hume, W. Giles, and A. M. Brown. 1981. Sodium current depression by lidocaine and quinidine in isolated ventricular cells. Nature (Lond.). 291:325-327.

30. Grant, A. O., J. L. Trantham, K. K. Brown, and H. C. Strauss. 1982 $\mathrm{pH}$-dependent effects of QUIN on the kinetics of $\mathrm{dV} / \mathrm{dt}$ max in guinea pig ventricular myocardium. Circ. Res. 50:369-376.

31. Jazayeri, M. R., G. VanWhye, B. Avitall, J. McKinnie, P. Tchou, and M. Akhtar. 1989. Isoproterenol reversal of antiarrhythmic effects in patients with inducible sustained ventricular tachyarrhythmias. J. Am. Coll. Cardiol. 14:705711.

32. Avitall, B., J. W. Hare, P. Tchou, M. Jazayeri, and M. Akhtar. 1991 Flecainide toxicity: reversal of drug effects by isoproterenol infusion. $J$. Cardiovasc. Electrophysiol. 2:431-440.

33. Morady, F., W. H. Kou, A. H. Kadish, S. D. Nelson, L. K. Toivonen, J. A Kushner, S. Schmaltz, and M. DeBuitler. 1988. Antagonism of quinidine's elec trophysiologic effects by epinephrine in patients with ventricular tachycardia. $J$ Am. Coll. Card. 12:388-394.

34. Schwarz, W., P. T. Palade, and B. Hille. 1977. Local anesthetics: effect of $\mathrm{pH}$ on use-dependent block of sodium channels in frog muscle. Biophys. $J$. 20:343-368.

35. Dominguez, G., and H. A. Fozzard. 1979. Effect of stretch on conduction velocity and cable properties of cardiac Purkinje fibers. Am. J. Physiol. 237:C119-C124

36. Calkins, H., W. L. Maughan, D. A. Kass, K. Sagawa, and J. H. Levine 1989. Electrophysiological effect of volume load in isolated canine hearts. Am.J. Physiol. 256:H1697-H1706.

37. Gornick, C. C., H. G. Tobler, M. C. Pritzker, I. C. Tuna, A. Almquist, and D. G. Benditt. 1986. Electrophysiologic effects of papillary muscle traction in the intact heart. Circulation. 73:1013-1021.

38. Franz, M. R., D. Burkhoff, D. T. Yue, and K. Sagawa. 1989. Mechanically induced action potential changes and arrhythmia in isolated and in situ canine hearts. Cardiovasc. Res. 23:213-223.

39. Hansen, D. E., C. S. Craig, and L. M. Hondeghem. 1990. Stretch-induced arrhythmias in the isolated canine ventricle: evidence for the importance of mechanoelectrical feedback. Circulation. 81:1094-1105.39.

40. Tande, P. M., E. Mortensen, and H. Refsum. 1991. Rate-dependent differences in dog epi- and endocardial monophasic action potential configuration in vivo. Am. J. Physiol. 261:H1387-H1391.
41. Franz, M. R., and A. Costard. 1988. Frequency-dependent effects of quinidine on the relationship between action potential duration and refractoriness in the canine heart in situ. Circulation. 77:1177-1184.

42. Nademanee, K. W., G. Stevenson, J. N. Weiss, V. B. Frame, M. G. Antimisiaris, T. Suithichaiyakul, and C. M. Pruitt. 1990. Frequency-dependent effects of quinidine on the ventricular action potential and QRS duration in humans. Circulation. 81:790-796.

43. Litovsky, S. H., and C. Antzelevitch. 1989. Rate dependence of action potential duration and refractoriness in canine ventricular endocardium differs from that of epicardium: the role of transient outward current. J. Am. Coll. Cardiol. 14:1053-1066.

44. Imaizumi, Y., and W. R. Giles. 1987. Quinidine-induced inhibition of transient outward current in cardiac muscle. Am. J. Physiol. 253:H704-H708.

45. Litovsky, S. H., and C. Antzelevitch. 1988. Transient outward current prominent in canine ventricular epicardium but not endocardium. Circ. Res. 62:116-126.

46. Furukawa, T., R. J. Myerburg, N. Furukawa, A. L. Bassett, and S. Kimura. 1990. Differences in transient outward currents of feline endocardial and epicardial myocytes. Circ. Res. 67:1287-1291.

47. Fedida, D., and W. R. Giles. 1991. Regional variations in action potentials and transient outward current in myocytes isolated from rabbit left ventricle. $J$. Physiol. (Lond.). 442:191-209.

48. Peon, J., G. R. Ferrier, and G. K. Moe. 1978. The relationship of excitability to conduction velocity in canine Purkinje tissue. Circ. Res. 43:125-135.

49. Nattel, S. 1987. Interval-dependent effects of lidocaine on conduction in canine cardiac Purkinje fibers: experimental observations and theoretical analysis. J. Pharmacol. Exp. Ther. 241:275-281.

50. Johns, J. A., Anno, T., P. B. Bennett, D. J. Snyuders, and L. M. Hondeghem. 1989. Temperature and voltage dependence of sodium channel blocking and unblocking by 0 -demethyl encainide in isolated guinea pig myocytes. $J$. Cardiovasc. Pharmacol. 13:826-835.

51. Sheets, M. F., D. A. Hanck, and H. A. Fozzard. 1988. Nonlinear relation between $V_{\max }$ and $l_{\mathrm{Na}}$ in canine cardiac Purkinje cells. Circ. Res. 63:386-398.

52. Cohen, C. J., B. P. Bean, and R. W. Tsien. 1984. Maximal upstroke velocity as an index of available sodium conductance. Circ. Res. 54:636-651.

53. Courtney, K. R. 1987. Quantitative structure/activity relations based on use-dependent block and repriming kinetics in myocardium. J. Mol. Cell. Cardiol. 19:319-330. 